Review Article

\title{
Energy Management for Energy Harvesting-Based Embedded Systems: A Systematic Mapping Study
}

\author{
Xingyu Miao $\mathbb{i}$ and Yongqi Ge $\mathbb{i}$ \\ School of Information Engineering, Ningxia University, Yinchuan 750021, China \\ Correspondence should be addressed to Yongqi Ge; geyongqi@nxu.edu.cn
}

Received 6 July 2020; Accepted 16 October 2020; Published 30 October 2020

Academic Editor: Fabio Massaro

Copyright (c) 2020 Xingyu Miao and Yongqi Ge. This is an open access article distributed under the Creative Commons Attribution License, which permits unrestricted use, distribution, and reproduction in any medium, provided the original work is properly cited.

\begin{abstract}
Energy management for energy harvesting-based embedded systems (EHES) is an emerging field, which aims to collect renewable energy from the environment to power an embedded system. In this work, we use the systematic mapping method to study the relevant literature, with the objective of exploring and analysing the state of the art in energy management for EHES, as well as to provide assistance for subsequent literature reviews. To this end, we conducted extensive searches to find articles related to energy harvesting, embedded systems, energy consumption, and energy management. We searched for papers from January 2005 to July 2019 from three mainstream databases, ACM, IEEE Xplore, and Web of Science, and found more than 3000 papers about EHES. Finally, we selected 142 eligible papers. We have completed the system mapping research from five aspects, namely, (1) research type (validation research, evaluation research, solution proposal, philosophical paper, opinion, and experience), (2) research goals (application or theory), (3) application scenarios, (4) tools or methods, and (5) paper distribution, such as publication year and authors' nationality. The results showed that the major research type of the EHES papers is validation research, accounting for $65 \%$, which indicated research is still in the theoretical stage and many researchers focus on how to improve the efficiency of harvesting energy, develop a reasonable energy supply plan, and adapt EHES for real-world requirements. Furthermore, this work reviews the tools used for EHES. As the future development direction, it is indispensable to provide tools to EHES for research, testing, development, and so on. The results of our analysis provide significant contributions to understanding the existing knowledge and highlighting potential future research opportunities in the EHES field.
\end{abstract}

\section{Introduction}

With an increase of network speed and hardware computing capabilities, many recent studies have focused on IoT and AI. The number of intelligent terminals, which are essential for the Internet of Things and artificial intelligence, has risen rapidly $[1,2]$. Most terminal devices are battery-powered. Therefore, the arbitrary use of such devices will reduce battery life which, in turn, decides the terminal life. In this situation, some solutions have been proposed to reduce energy consumption, to a certain extent, such as dynamic voltage and frequency scaling (DVFS) [3-5] and dynamic power management [6-8]. In some cases, it is difficult to recharge or change the battery; for example, in the biomedical field, implants are mainly powered by batteries with capacities limited by physical size constraints. One method to address this issue is to use rechargeable batteries. The most common recharging method used in medical devices utilizes inductive coupling, but this has disadvantages; for example, when the user needs to charge, they must place the external charging device very close to the implanted device $[9,10]$. In order to address this issue, researchers have used the renewable energy of the surrounding environment to harvest energy (e.g., a photovoltaic array embedded under the eye's conjunctiva coverts incident solar energy into electrical energy to be stored in a battery [10]). Energy harvesting technologies can be used to power embedded devices and replenish the energy of batteries in embedded devices, thus extending their running time. An embedded system with energy harvesting technology is referred to as EHES. An 
EHES converts renewable energy into electricity to power the system. Due to the unpredictability of the environment (e.g., a period of overcast/rain, or solar power not being available during the night), the harvesting of energy is also uncertain. As shown in Figure 1, if the energy available from a renewable source is higher than the required energy of a system, then the system can function without any energy from the battery. If the situation is exactly the opposite, as shown by the shaded regions in Figure 1, additional energy needs to be extracted from the battery to ensure the sustained operation of the embedded system. Moreover, some power strategies, task scheduling strategies, and battery storage strategies are imperfect, resulting in considerable energy consumption of the devices which, then, need frequent charging. This indicates the energy consumption and energy storage of EHES is the main problem in the future development of this domain. Compared with batterypowered embedded devices, energy harvesting embedded devices collect renewable energy from the ambient environment, which can effectively eliminate the demand for battery replacement.

In order to summarize the research status of a special research field and explore the researchable directions within it, a literature review is required. However, researchers in the field of software engineering (SE) usually do not follow a systematic approach when conducting literature research. At present, several guidelines have been proposed to conduct literature research, such as systematic mapping study (SMS) or systematic literature review (SLR) [12-17]. The SMS provides a superficial overview of a certain field, such as the number of research works published in the field and their classification, which is usually done graphically. An SMS helps the reviewer to find research topics in specific fields, as well as contributing to better follow-up research such as SLR. Compared with SMS, SLR can conduct further literature research through an in-depth analysis of selected works. Therefore, SLR is an extension of SMS. In this work, we use SMS with a focus on providing an overview of EHES, identifying research evidence for this topic, and presenting mainly quantitative results $[13,14]$. We conducted this work to answer five different research questions about EHES and to collect relevant research by snowballing [18]. We classified the research based on research types, research objectives, application scenarios, tools, methods, and publication venues and collected the required data by reading titles, abstracts, keywords, and full texts. Considering that energy management is significant for EHES, it is necessary to conduct a systematic analysis to develop a comprehensive understanding for this topic's research and practice. As far as we know, there currently exist no literature reviews which fill this gap. This work, therefore, provides researchers with a study of the practices within this domain over the past fifteen years.

We focus on exploring and analysing the state of the art in energy management for EHES. The remainder of this paper is organized as follows: the SMS is described in Section 2. In Section 3, we present the study results and answer the research questions. And in Section 4, we present the future

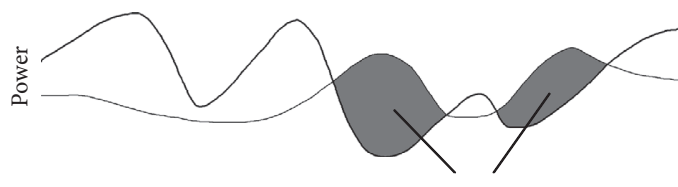

Energy required from grid on battery (unsustainable operation) Time

— Power produced by external sources

Figure 1: Profile of energy required and energy available from external sources. The unsustainable operation can be caused by the imbalance of available and required power, in which case additional energy needs to be extracted from the battery [11].

challenges of the EHES. Finally, we conclude the work in Section 5 .

\section{The Systematic Mapping Processes}

In this section, we follow the systematic mapping process, as defined in the guidelines provided by Petersen et al. $[13,14]$. As shown in Figure 2, there are five steps in the SMS. First, the review scope is obtained by the definition of the research question. Second, a search is conducted to find all papers within this scope. Third, relevant papers are found in this field by screening the found papers. Then, by reading the abstracts and looking for keywords and concepts which reflect the contributions of the papers, they are combined to form an understanding of the nature and contributions of the research, thus forming a classification scheme. Finally, with the abovementioned classification scheme, relevant papers are classified in the scheme, and data are extracted. Using the data to analyse each category, the systematic map is presented by means of mapping.

2.1. Research Questions. The overall goal of this SMS is to determine the research type, research objectives, application scenarios, tools, methods, and publications relating to EHES since 2005. This objective led to the following questions:

(i) RQ1: what type of research has been carried out in the research period and how has it developed?

Rationale: popular research types can provide researchers with research trends and future forms of development in the field, as well as which directions provide open areas for research. Our focus is to find the most popular research trends in these years by studying these studies.

(ii) RQ2: what are the research goals?

Rationale: our focus is to group selected studies, according to different research objectives.

(iii) RQ3: what are the application scenarios for the energy management of EHES?

Rationale: our focus is to find popular application scenarios for the energy management of EHES. 

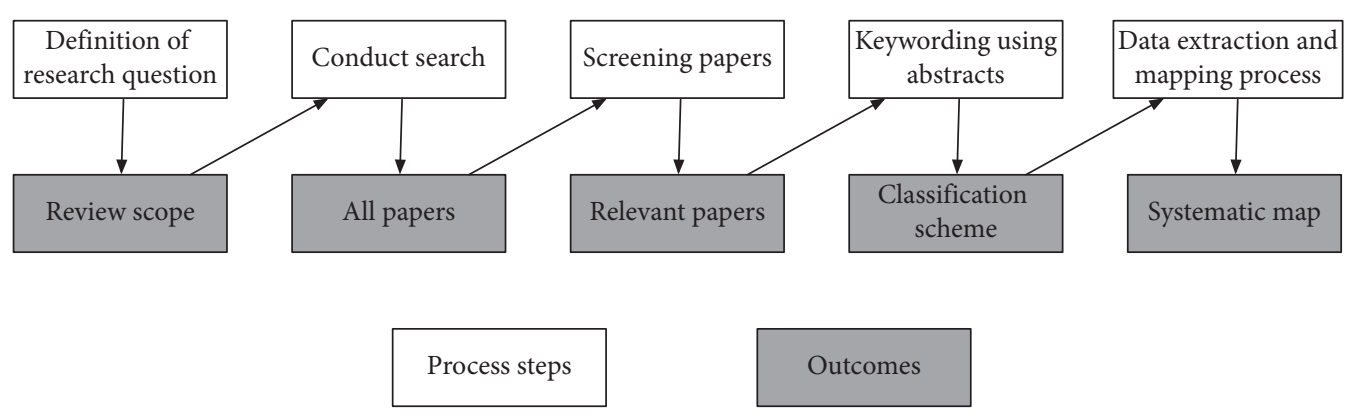

FIGURE 2: The systematic mapping study.

(iv) RQ4: how many tool papers and method papers were proposed?

Rationale: our aim is to find out the tool and method papers which can be used by other researchers in their studies.

(v) RQ5: how are the papers distributed?

Rationale: our focus is to find the publication year, type of publication, and author information for the papers.

2.2. Study Collection and Study Screening. In the process of search and selection, we will encounter some problems:

(1) How to search?

First, we needed to select the approximate database range and find the query rules for searching the databases. Then, we determined a query sentence and, finally, conducted the preliminary search. The trial search helped us to find appropriate search databases and search methods. As different databases employ search engines with different search capabilities, we needed a generic query statement, for example, which was expressed as "(embedded systems) AND (energy harvesting)"; however, searching with this method did not allow us to determine whether the papers were what we needed. Therefore, we required further screening.

(2) How to select?

Usually, if reading the title does not indicate the core meaning of the paper, it is necessary to read the abstract of the paper. In some cases (e.g., after reading the title and abstract, we still did not understand the core meaning of the paper), we were required to read the full text or conclusions. In the formal selection, we needed to formulate selection criteria.

2.2.1. Detailed Process. The search and selection process had four steps, as shown in Figure 3.

(i) Step 1: searching for papers in databases. We chose three relatively popular databases for our subject field (see Table 1) and searched for relevant papers using the query statement.

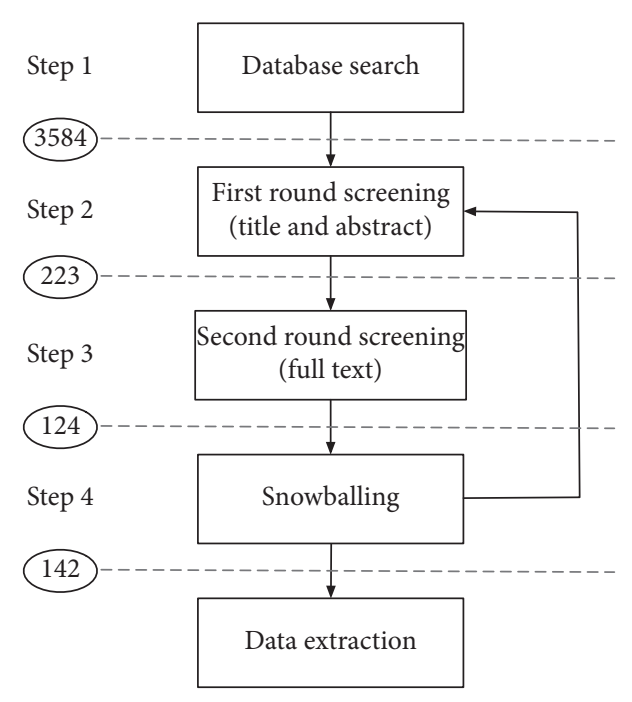

Figure 3: Formal search and selection process.

(ii) Step 2: conducting the first round of screening by reading the titles and abstracts of the results of Step 1 search.

(iii) Step 3: if only reading the title and abstract did not indicate the screened paper's core meaning in Step 2 , then we read the full text.

(iv) Step 4: using the method of snowballing [18] to screen the references in the results of Step 3. Then, we repeated Steps 2-4 for the screened papers.

2.2.2. Search Scope. We used three authoritative and wellknown databases in our field-IEEE, ACM, and WOS-for this paper collection. Google Scholar was not included in the database search, as its search results may have considerable overlap with other databases and it had insufficient accuracy for our purposes. The research period was set from January 2005 to July 2019. As far as we know, no paper exists which can serve as milestone in this domain, as this topic is broad and extensive. Therefore, considering that 15 years is a reasonable time for systematic mapping research, we started our search in 2005 .

2.2.3. Search Query. To conduct a SMS, building a search string is very important, and thus, we used the PICO 
TABle 1: Database.

\begin{tabular}{lcc}
\hline Database & Link & Matched scope in database \\
\hline IEEE Xplore & https://ieeexplore.ieee.org/Xplore/guesthome.jsp & Title, abstract \\
ACM Digital Library & https://libraries.acm.org & Title, abstract, keywords \\
Web of Science & http://login.webofknowledge.com & Title, abstract, keywords \\
\hline
\end{tabular}

(Population, Interventions, Comparison, and Outcomes) model proposed by Petersen et al. [14] to build the search string, where the population is a specific SE role, type, or application area; interventions are software technologies or methodologies to address specific issues in SE; comparison determines the technologies, techniques, tools, methods, or strategies to be extracted and compared; and outcomes are the influencing factors of the intervention on the practitioners. As shown in Table 2, for each component of the PICO model, we determined many sentences that described the learning topic. We used the guidelines proposed by Petersen et al. [14] to extract keywords. Table 3 shows the keywords extracted with the PICO model.

Boolean "OR" is used to connect synonyms and Boolean "AND" is used to connect terms. The statement of our query was expressed as "(design OR development) AND (embedded systems) AND (energy OR harvesting OR energy harvesting) AND (energy storage) AND (energy efficiency) AND (application OR tool OR method) AND (energy management OR life)."

2.2.4. Search Criteria. In the guidelines of Petersen et al. [13], they pointed out that it is important to determine whether the study meets the necessary characteristics in order to be included or excluded from the SMS. Using this guide as a basis, the following criteria for inclusion and exclusion should be considered when selecting papers:

Inclusion criteria:

(i) The paper offers a new approach or improvements for the energy management of EHES (e.g., energy consumption, use of energy, and collection of energy)

Exclusion criteria:

(i) The paper only has an abstract without full text

(ii) The paper is a poster or book

(iii) The paper only mentions the term energy harvesting

(iv) The paper is not in English

(v) The paper only introduces a new collection method which does not specify its energy management

(vi) The paper only introduces a new energy harvesting embedded system

2.3. Study Classification. In this section, we followed the guidelines provided by Petersen et al. [13]. We used abstracts and keywords from collection-related research to classify research. In this work, we have classified the research into five different categories depending on the research questions. The taxonomy of the related article is shown in Figure 4.

2.3.1. Research Types Classification. We chose an existing classification framework by Wieringa et al. [19] to classify the selected papers. The categories do not require a detailed evaluation of each paper and, thus, are easy to interpret and classify. For example, for research works that were not implemented in practice, evaluation research can be excluded. Moreover, validation research can easily be determined, which only requires checking whether the paper states hypotheses or experimental design is a main component of the paper. In addition, the framework also classifies study into solution proposal paper, philosophical papers, opinion papers, and experience papers:

(i) A validation research paper is a paper which was not implemented in practice, instead of addressing issues through lab experiments, analysis, and design.

(ii) An evaluation research paper evaluates research works that were implemented in practice, indicating the advantages and disadvantages of these research works in the implementation process and the final result. Usually, industrial studies fall into this category.

(iii) A solution proposal paper provides an improvement to an existing solution or proposes a new technology.

(iv) A philosophical paper introduces a new way of looking at existing things or a new conceptual framework.

(v) An opinion paper provides someone's personal opinion on technology, tool, or method.

(vi) An experience paper details an author's personal experience of technology, tool, or method.

2.3.2. Research Goals Classification. We identified the overall goals of the research and divided them into the following four categories:

(i) Energy harvesting and conversion strategies for EHES

(ii) Task scheduling strategies for EHES

(iii) Better strategies for battery storage to maintain battery life

(iv) Using EHES independently by the use of energy harvesting technology 
TABle 2: Description of our PICO model which matches the EHES.

\begin{tabular}{lc}
\hline Component & Description \\
$\begin{array}{l}\text { Population } \\
\text { Interventions }\end{array}$ & $\begin{array}{c}\text { Eethod design and development of energy storage efficiency; design and development of methods to reduce energy } \\
\text { consumption }\end{array}$ \\
$\begin{array}{c}\text { Comparison } \\
\text { Outcomes }\end{array}$ & $\begin{array}{c}\text { We identify different applications and tools of EHES, analyse different methods and technologies for energy management, } \\
\text { and compare research on EHES }\end{array}$ \\
\hline
\end{tabular}

TABle 3: Extracted keywords from the PICO model.

\begin{tabular}{|c|c|}
\hline Component & Keywords \\
\hline Population & $\begin{array}{c}\text { Energy harvesting, embedded system, energy } \\
\text { management }\end{array}$ \\
\hline Interventions & $\begin{array}{c}\text { Method, design, development, energy storage, } \\
\text { efficiency, consumption }\end{array}$ \\
\hline Comparison & $\begin{array}{l}\text { Applications, tools, analysis, methods, } \\
\text { technologies, energy management }\end{array}$ \\
\hline Outcomes & Life, energy management, methods \\
\hline
\end{tabular}

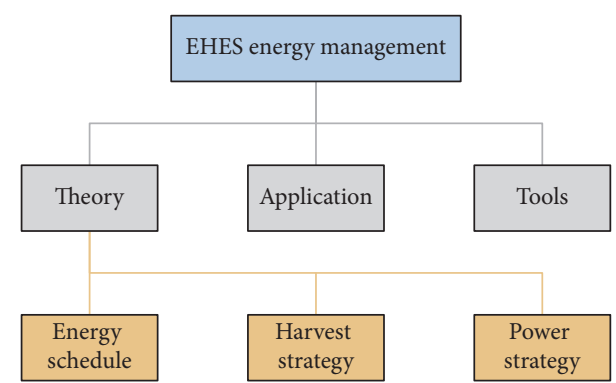

Figure 4: Taxonomy of research literature on EHES energy management.

2.3.3. Application Scenarios Classification. We identified the following application scenarios, based on all studies:

(i) Building: the EHES provides energy for building or road structure health monitoring systems

(ii) Medical: the energy harvesting embedded system can power some devices which are transplanted in the human body

(iii) Machine: the energy harvesting embedded system can provide energy for machine component health monitoring or power for certain control systems

(iv) Wearable equipment: the collection of vibrational energy, thermal energy, and so on can be converted into electrical energy for monitoring systems of certain wearable equipment or for the life of the equipment

2.3.4. Tool Papers and Method Papers Classification. Papers are divided into energy management tool papers and papers that can effectively improve energy consumption, energy harvesting, and energy storage technologies or improve on existing methods to improve efficiency.
2.3.5. Distribution Classification. We used a general classification scheme for publication venue types, publishers, publication year, and authors' nationality. Usually, venue categories are divided into international journals, international conferences, seminars, and others.

2.4. Data Extraction and Study Mapping. We answered the research questions presented above and used an Excel table to extract data from each selected study. The form fields included the research type to answer RQ1; research goals to answer RQ2; application scenario details (if any) to answer RQ3; method and tool details (if any) to answer RQ4; and year of publication, publication venue, and publisher to answer RQ5. After reading each research paper, we filled out the data form. The specific extraction results are shown in Table 4. Finally, the research was mapped to the above classification definitions, and the research frequency under each classification was visualized in the form of a graph.

\section{Result}

3.1. Search Selection and Snowballing Results. As shown in Figure 3, 3584 papers were retrieved from the three databases during the database search. After the first round of screening, 223 papers were retained. After the second round of screening, 124 papers were retained and 18 papers were determined by snowballing [18]. Finally, a total of 142 papers were selected.

3.2. RQ1: What Type of Research Has Been Carried Out in the Research Period and How Has It Developed? Our focus was to discover the popular research types in 2005-2019. As shown in Figure 3, the most frequent research type was validation research, accounting for 65\% of papers (92 out of 142), followed by solution proposals, accounting for $22 \%$ of papers (31 out of 142); 12\% (18 out of 142) of research papers were evaluation research, and 1\% (1 out of 142) were experience papers. No opinion or philosophical papers were found.

As shown in Figure 5, the number of validation research studies published since 2009 has increased, and it is important to note that more than $50 \%$ of published papers were in this category. This shows the importance of energy management for EHES in lab experiments. Meanwhile, the number of solution proposals, as shown in Figure 6, was not much; this indicates that most technologies have not been implemented in practice. The articles of validation research and solution proposal occupied $87 \%$ of the research types and, so, researchers can easily select papers to conduct SLR. 
TABLE 4: Data extracted from the selected papers.

\begin{tabular}{|c|c|c|c|c|c|c|c|c|}
\hline ID & Ref & $\begin{array}{c}\text { RQ1 } \\
\text { Research type }\end{array}$ & $\begin{array}{c}\text { RQ2 } \\
\text { Research goal }\end{array}$ & $\begin{array}{c}\text { RQ3 } \\
\text { Application scenario }\end{array}$ & $\begin{array}{c}\text { RQ4 } \\
\text { Tool or method }\end{array}$ & $\begin{array}{l}\text { RQ5 } \\
\text { Year }\end{array}$ & Publish type & Publisher \\
\hline 1 & {$[20]$} & Validation research & Application & - & Tool & 2019 & Journal & Others \\
\hline 2 & {$[21]$} & Evaluation research & Power strategy & - & Method & 2019 & Journal & Springer \\
\hline 3 & {$[22]$} & Validation research & Energy schedule & - & - & 2019 & Journal & IEEE \\
\hline 4 & [23] & Validation research & Energy schedule & - & Method & 2019 & Conferences & $\mathrm{ACM}$ \\
\hline 5 & {$[24]$} & Evaluation research & Energy schedule & - & Method & 2019 & Journal & $\mathrm{ACM}$ \\
\hline 6 & [25] & Solution proposal & Application & Building & - & 2019 & Journal & Others \\
\hline 7 & {$[26]$} & Solution proposal & Application & - & - & 2019 & Journal & Others \\
\hline 8 & [27] & Solution proposal & Application & Machine & - & 2018 & Journal & Wiley \\
\hline 9 & {$[28]$} & Validation research & Application & Machine & - & 2018 & Conferences & IEEE \\
\hline 10 & [29] & Solution proposal & Harvest & strategy & Method & 2018 & Conferences & $\mathrm{ACM}$ \\
\hline 11 & {$[30]$} & Validation research & Application & - & Tool & 2018 & Conferences & $\mathrm{ACM}$ \\
\hline 12 & {$[31]$} & Validation research & Application & Wearable equipment & - & 2018 & Symposium & IEEE \\
\hline 13 & {$[32]$} & Experience papers & Application & $L^{1-1}$ & - & 2018 & Conferences & IEEE \\
\hline 14 & {$[33]$} & Validation research & Application & Machine & - & 2018 & Journal & IEEE \\
\hline 15 & {$[34]$} & Validation research & Application & Building & - & 2018 & Conferences & Springer \\
\hline 16 & {$[35]$} & Validation research & Energy schedule & - & Method & 2018 & Conferences & IEEE \\
\hline 17 & {$[36]$} & Solution proposal & Energy schedule & - & Method & 2018 & Conferences & IEEE \\
\hline 18 & {$[37]$} & Validation research & Application & Machine & - & 2018 & Conferences & IEEE \\
\hline 19 & {$[38]$} & Evaluation research & Harvest strategy & Machine & - & 2018 & Journal & Others \\
\hline 20 & {$[39]$} & Validation research & Harvest strategy & - & - & 2018 & Journal & Others \\
\hline 21 & {$[40]$} & Solution proposal & Application & Wearable equipment & Method & 2017 & Conferences & $\mathrm{ACM}$ \\
\hline 22 & {$[41]$} & Solution proposal & Application & Machine & Method & 2017 & Conferences & IEEE \\
\hline 23 & {$[42]$} & Validation research & Power strategy & - & Tool & 2017 & Conferences & IEEE \\
\hline 24 & {$[43]$} & Validation research & Energy schedule & - & Method & 2017 & Journal & Others \\
\hline 25 & {$[44]$} & Validation research & Energy schedule & - & Method & 2017 & Conferences & IEEE \\
\hline 26 & {$[45]$} & Validation research & Power strategy & - & Method & 2017 & Journal & $\mathrm{ACM}$ \\
\hline 27 & {$[46]$} & Evaluation research & Energy schedule & - & Method & 2017 & Conferences & IEEE \\
\hline 28 & {$[47]$} & Solution proposal & Harvest strategy & - & - & 2017 & Journal & Others \\
\hline 29 & {$[48]$} & Validation research & Application & Machine & - & 2016 & Journal & Others \\
\hline 30 & [49] & Solution proposal & Application & Medical & - & 2016 & Conferences & Others \\
\hline 31 & {$[50]$} & Validation research & Application & Building & Method & 2016 & Journal & Others \\
\hline 32 & {$[51]$} & Validation research & Power strategy & - & Method & 2016 & Conferences & IEEE \\
\hline 33 & {$[52]$} & Validation research & Energy schedule & - & Method & 2016 & Journal & Others \\
\hline 34 & {$[53]$} & Validation research & Energy schedule & - & Method & 2016 & Journal & Others \\
\hline 35 & {$[54]$} & Validation research & Harvest strategy & - & Method & 2015 & Conferences & IEEE \\
\hline 36 & {$[55]$} & Validation research & Energy schedule & - & Method & 2015 & Conferences & IEEE \\
\hline 37 & {$[56]$} & Validation research & Harvest strategy & - & Method & 2015 & Conferences & $\mathrm{ACM}$ \\
\hline 38 & {$[57]$} & Validation research & Application & Wearable equipment & Method & 2015 & Conferences & IEEE \\
\hline 39 & {$[10]$} & Validation research & Application & Medical & - & 2015 & Conferences & IEEE \\
\hline 40 & {$[58]$} & Validation research & Energy schedule & - & Method & 2015 & Conferences & IEEE \\
\hline 41 & {$[59]$} & Validation research & Energy schedule & - & Method & 2015 & Conferences & IEEE \\
\hline 42 & {$[60]$} & Solution proposal & Energy schedule & - & Method & 2015 & Journal & IEEE \\
\hline 43 & {$[61]$} & Validation research & Application & Wearable equipment & - & 2015 & Journal & IEEE \\
\hline 44 & {$[62]$} & Validation research & Energy schedule & - & Method & 2014 & Conferences & $\mathrm{ACM}$ \\
\hline 45 & {$[63]$} & Validation research & Energy schedule & - & Method & 2014 & Conferences & IEEE \\
\hline 46 & {$[64]$} & Validation research & Energy schedule & - & Method & 2014 & Journal & IEEE \\
\hline 47 & {$[65]$} & Solution proposal & Energy schedule & - & Method & 2014 & Journal & IEEE \\
\hline 48 & {$[66]$} & Validation research & Application & Wearable equipment & - & 2014 & Conferences & IEEE \\
\hline 49 & {$[67]$} & Validation research & Harvest strategy & - & Tool & 2014 & Conferences & $\mathrm{ACM}$ \\
\hline 50 & {$[68]$} & Evaluation research & Harvest strategy & - & Method & 2014 & Journal & IEEE \\
\hline 51 & [69] & Validation research & Application & Building & - & 2014 & Conferences & Others \\
\hline 52 & {$[70]$} & Validation research & Harvest strategy & - & Method & 2014 & Conferences & IEEE \\
\hline 53 & {$[71]$} & Solution proposal & Energy schedule & - & Method & 2014 & Symposium & $\mathrm{ACM}$ \\
\hline 54 & {$[72]$} & Solution proposal & Application & Wearable equipment & - & 2014 & Journal & Others \\
\hline 55 & {$[73]$} & Evaluation research & Application & Building & - & 2014 & Journal & Springer \\
\hline 56 & {$[74]$} & Validation research & Energy schedule & - & - & 2014 & Journal & IEEE \\
\hline 57 & {$[5]$} & Validation research & Energy schedule & - & Method & 2013 & Symposium & IEEE \\
\hline 58 & {$[75]$} & Solution proposal & Energy schedule & - & Method & 2013 & Conferences & IEEE \\
\hline 59 & {$[76]$} & Validation research & Application & Machine & - & 2013 & Conferences & Others \\
\hline
\end{tabular}


TABle 4: Continued.

\begin{tabular}{|c|c|c|c|c|c|c|c|c|}
\hline ID & Ref & $\begin{array}{c}\text { RQ1 } \\
\text { Research type }\end{array}$ & $\begin{array}{c}\text { RQ2 } \\
\text { Research goal }\end{array}$ & $\begin{array}{c}\text { RQ3 } \\
\text { Application scenario }\end{array}$ & $\begin{array}{c}\text { RQ4 } \\
\text { Tool or method }\end{array}$ & $\begin{array}{l}\text { RQ5 } \\
\text { Year }\end{array}$ & Publish type & Publisher \\
\hline 60 & [77] & Evaluation research & Application & Machine & Tool & 2013 & Journal & ACM \\
\hline 61 & {$[78]$} & Validation research & Application & Building & - & 2013 & Workshop & IEEE \\
\hline 62 & [79] & Validation research & Harvest strategy & - & Method & 2013 & Journal & Others \\
\hline 63 & {$[80]$} & Validation research & Power strategy & - & Method & 2013 & Journal & Others \\
\hline 64 & {$[81]$} & Validation research & Energy schedule & - & - & 2013 & Journal & Others \\
\hline 65 & {$[82]$} & Validation research & Application & Building & - & 2013 & Journal & Others \\
\hline 66 & {$[83]$} & Evaluation research & Application & Machine & - & 2013 & Journal & Others \\
\hline 67 & [84] & Validation research & Application & Building & - & 2013 & Conferences & IEEE \\
\hline 68 & {$[85]$} & Solution proposal & Harvest strategy & - & Method & 2013 & Workshop & $\mathrm{ACM}$ \\
\hline 69 & {$[86]$} & Validation research & Application & Medical & - & 2013 & Journal & Others \\
\hline 70 & [87] & Evaluation research & Power strategy & - & Method & 2013 & Workshop & $\mathrm{ACM}$ \\
\hline 71 & {$[88]$} & Validation research & Harvest strategy & - & - & 2013 & Conferences & IEEE \\
\hline 72 & [89] & Evaluation research & Harvest strategy & - & Method & 2013 & Conferences & IEEE \\
\hline 73 & {$[90]$} & Validation research & Application & Building & - & 2013 & Journal & Others \\
\hline 74 & {$[91]$} & Solution proposal & Harvest strategy & - & Method & 2013 & Journal & $\mathrm{ACM}$ \\
\hline 75 & {$[92]$} & Validation research & Application & - & Method & 2012 & Journal & Springer \\
\hline 76 & [93] & Validation research & Energy schedule & - & Method & 2012 & Conferences & IEEE \\
\hline 77 & {$[94]$} & Solution proposal & Energy schedule & - & Method & 2012 & Conferences & IEEE \\
\hline 78 & [95] & Validation research & Application & Medical & Tool & 2012 & Conferences & IEEE \\
\hline 79 & {$[96]$} & Validation research & Application & Machine & Tool & 2012 & Journal & Others \\
\hline 80 & [97] & Validation research & Energy schedule & - & Method & 2012 & Conferences & IEEE \\
\hline 81 & {$[98]$} & Evaluation research & Harvest strategy & - & Method & 2012 & Conferences & $\mathrm{ACM}$ \\
\hline 82 & [99] & Validation research & Harvest strategy & - & Method & 2012 & Journal & Others \\
\hline 83 & {$[100]$} & Evaluation research & Harvest strategy & - & Method & 2012 & Journal & IEEE \\
\hline 84 & {$[101]$} & Validation research & Application & - & - & 2012 & Conferences & IEEE \\
\hline 85 & {$[102]$} & Validation research & Energy schedule & - & Method & 2012 & Journal & IEEE \\
\hline 86 & {$[103]$} & Validation research & Energy schedule & - & Method & 2011 & Conferences & IEEE \\
\hline 87 & {$[104]$} & Validation research & Energy schedule & - & Method & 2011 & Journal & Others \\
\hline 88 & {$[105]$} & Validation research & Energy schedule & - & Method & 2011 & Conferences & IEEE \\
\hline 89 & {$[106]$} & Validation research & Application & Wearable equipment & - & 2011 & Journal & Others \\
\hline 90 & {$[107]$} & Solution proposal & Energy schedule & - & Method & 2011 & Conferences & $\mathrm{ACM}$ \\
\hline 91 & {$[108]$} & Validation research & Harvest strategy & - & Method & 2011 & Workshop & IEEE \\
\hline 92 & [109] & Validation research & Energy schedule & - & Method & 2011 & Journal & Others \\
\hline 93 & {$[110]$} & Validation research & Harvest strategy & - & Method & 2011 & Conferences & IEEE \\
\hline 94 & {$[111]$} & Solution proposal & Energy schedule & - & Method & 2011 & Conferences & IEEE \\
\hline 95 & {$[112]$} & Validation research & Application & - & Tool & 2011 & Journal & IEEE \\
\hline 96 & [113] & Solution proposal & Energy schedule & - & Method & 2011 & Conferences & Springer \\
\hline 97 & {$[114]$} & Evaluation research & Application & Building & - & 2011 & Symposium & IEEE \\
\hline 98 & {$[115]$} & Evaluation research & Application & Building & - & 2011 & Journal & Others \\
\hline 99 & {$[116]$} & Evaluation research & Harvest strategy & - & - & 2011 & Journal & Springer \\
\hline 100 & [117] & Validation research & Harvest strategy & - & - & 2010 & Conferences & $\mathrm{ACM}$ \\
\hline 101 & {$[118]$} & Validation research & Harvest strategy & - & Method & 2010 & Journal & Others \\
\hline 102 & {$[119]$} & Validation research & Application & Building & - & 2010 & Journal & Others \\
\hline 103 & {$[120]$} & Validation research & Application & Wearable equipment & - & 2010 & Conferences & IEEE \\
\hline 104 & {$[121]$} & Validation research & Energy schedule & -1 & Method & 2010 & Conferences & IEEE \\
\hline 105 & {$[122]$} & Validation research & Energy schedule & - & Method & 2010 & Journal & $\mathrm{ACM}$ \\
\hline 106 & {$[123]$} & Validation research & Application & Machine & - & 2010 & Workshop & $\mathrm{ACM}$ \\
\hline 107 & {$[124]$} & Validation research & Energy schedule & - & Method & 2010 & Journal & $\mathrm{ACM}$ \\
\hline 108 & {$[125]$} & Validation research & Energy schedule & - & Method & 2010 & Journal & Others \\
\hline 109 & {$[126]$} & Solution proposal & Harvest strategy & - & Method & 2010 & Conferences & IEEE \\
\hline 110 & {$[127]$} & Validation research & Application & Building & - & 2010 & Journal & $\mathrm{ACM}$ \\
\hline 111 & {$[128]$} & Validation research & Harvest strategy & - & Method & 2009 & Others & Others \\
\hline 112 & [129] & Validation research & Harvest strategy & - & Method & 2009 & Journal & Others \\
\hline 113 & {$[130]$} & Validation research & Harvest strategy & - & Method & 2009 & Journal & IEEE \\
\hline 114 & {$[131]$} & Validation research & Harvest strategy & - & Method & 2009 & Conferences & IEEE \\
\hline 115 & {$[132]$} & Validation research & Application & Machine & - & 2009 & Journal & IEEE \\
\hline 116 & [133] & Solution proposal & Energy schedule & - & Method & 2009 & Symposium & $\mathrm{ACM}$ \\
\hline 117 & {$[134]$} & Validation research & Application & Machine & - & 2009 & Conferences & IEEE \\
\hline 118 & {$[135]$} & Validation research & Energy schedule & - & Method & 2009 & Conferences & $\mathrm{ACM}$ \\
\hline 119 & [136] & Validation research & Harvest strategy & Building & Method & 2008 & Conferences & IEEE \\
\hline 120 & [137] & Solution proposal & Harvest strategy & - & Tool & 2008 & Conferences & IEEE \\
\hline
\end{tabular}


TABLE 4: Continued.

\begin{tabular}{|c|c|c|c|c|c|c|c|c|}
\hline ID & Ref & $\begin{array}{c}\text { RQ1 } \\
\text { Research type }\end{array}$ & $\begin{array}{c}\text { RQ2 } \\
\text { Research goal }\end{array}$ & $\begin{array}{c}\text { RQ3 } \\
\text { Application scenario }\end{array}$ & $\begin{array}{c}\text { RQ4 } \\
\text { Tool or method }\end{array}$ & $\begin{array}{l}\text { RQ5 } \\
\text { Year }\end{array}$ & Publish type & Publisher \\
\hline 121 & {$[138]$} & Solution proposal & Harvest strategy & - & Method & 2008 & Conferences & IEEE \\
\hline 122 & [139] & Solution proposal & Harvest strategy & - & Tool & 2008 & Journal & Others \\
\hline 123 & [140] & Validation research & Energy schedule & - & Method & 2008 & Conferences & IEEE \\
\hline 124 & {$[141]$} & Validation research & Application & Building & - & 2008 & Journal & Others \\
\hline 125 & [142] & Evaluation research & Harvest strategy & - & Method & 2008 & Conferences & Others \\
\hline 126 & [143] & Solution proposal & Harvest strategy & - & - & 2008 & Conferences & IEEE \\
\hline 127 & [144] & Validation research & Application & - & Method & 2008 & Conferences & IEEE \\
\hline 128 & [145] & Validation research & Application & Medical & Tool & 2007 & Symposium & IEEE \\
\hline 129 & [146] & Validation research & Application & - & Method & 2007 & Symposium & IEEE \\
\hline 130 & [147] & Solution proposal & Energy schedule & - & Method & 2007 & Journal & $\mathrm{ACM}$ \\
\hline 131 & [148] & Validation research & Application & Machine & - & 2007 & Journal & Others \\
\hline 132 & [149] & Validation research & Harvest strategy & - & Method & 2007 & Conferences & IEEE \\
\hline 133 & {$[150]$} & Validation research & Harvest strategy & - & Method & 2007 & Symposium & IEEE \\
\hline 134 & [151] & Solution proposal & Harvest strategy & - & Method & 2007 & Symposium & $\mathrm{ACM}$ \\
\hline 135 & [152] & Evaluation research & Energy schedule & - & Method & 2006 & Conferences & $\mathrm{ACM}$ \\
\hline 136 & [153] & Solution proposal & Harvest strategy & - & - & 2006 & Symposium & $\mathrm{ACM}$ \\
\hline 137 & [154] & Solution proposal & Application & Building & Method & 2005 & Conferences & AMC \\
\hline 138 & [155] & Validation research & Application & Wearable equipment & - & 2005 & Journal & IEEE \\
\hline 139 & [156] & Validation research & Energy schedule & $-^{1}$ & Method & 2005 & Conferences & $\mathrm{ACM}$ \\
\hline 140 & [157] & Validation research & Harvest strategy & - & Method & 2005 & Conferences & $\mathrm{ACM}$ \\
\hline 141 & [158] & Evaluation research & Harvest strategy & - & Tool & 2005 & Symposium & $\mathrm{ACM}$ \\
\hline 142 & [159] & Evaluation & Application & - & - & 2005 & Journal & IEEE \\
\hline
\end{tabular}

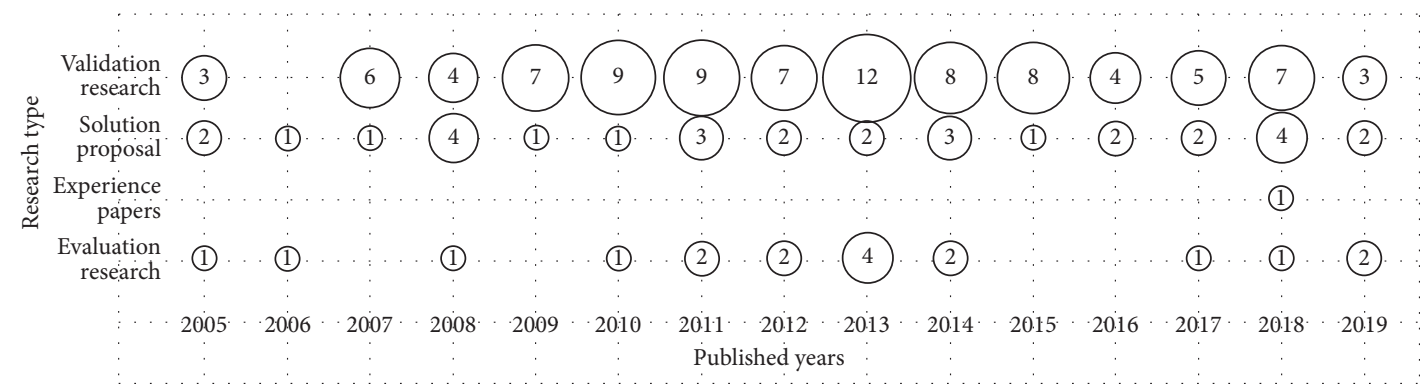

FIGURE 5: The number of research types per year.

There was only one solution proposal in 2005-2019, which was published in 2018, and there were no opinion or philosophical papers in these years. In the future, researchers need to provide more opinions, experience, and unique insights into this field. Future researchers need to apply ideas in practice to make the field more mature, like other fields.

3.3. RQ2: What Are the Research Goals? We identified four principal research goals, based on all selected studies. The first involved the method of collecting energy, which mainly studied novel methods to enhance the energy conversion rate or how to improve existing methods to enhance the energy conversion rate or increase energy harvesting efficiency. The second was about energy scheduling, involving how to effectively use the collected energy to supply the system to run and store power. The third was about battery strategies, which are important for the efficiency of battery storage. The fourth was about applications, focusing on using a variety of renewable energies to drive some sensors or support autonomous supply systems to achieve permanent operations.
As shown in Figure 7, 31\% of papers focused on energy scheduling (see, e.g., [52, 59, 62, 65, 97, 107]), such as studying how to combine energy-constrained scheduling algorithms with the introduction of an energy harvesting unit; $28 \%$ of papers focused on energy harvesting methods (see, e.g., [29]), such as using reinforcement learning (RL) to automatically configure a device to maximize energy storage or minimize energy consumption as much as possible (see, e.g., $[23,29,36,45])$. Since the energy harvesting by EHES is affected by geographic location and the surrounding environment, the collected data are different. If researchers use public datasets or publish data, the results may be replicated, but it is more difficult to replicate if they are not public. Unfortunately, since the authors in $[29,36,45]$ did not have public data, we could not fully replicate the results. Reference [23] requires a certain hardware foundation, we cannot build an experimental environment, and the results also cannot be replicated; $4 \%$ of papers considered power strategies (see, e.g., El Ghor and Chetto [160]), such as proposing an energy guarantee dynamic voltage and frequency scaling (EG-DVFS) algorithm which, compared with 


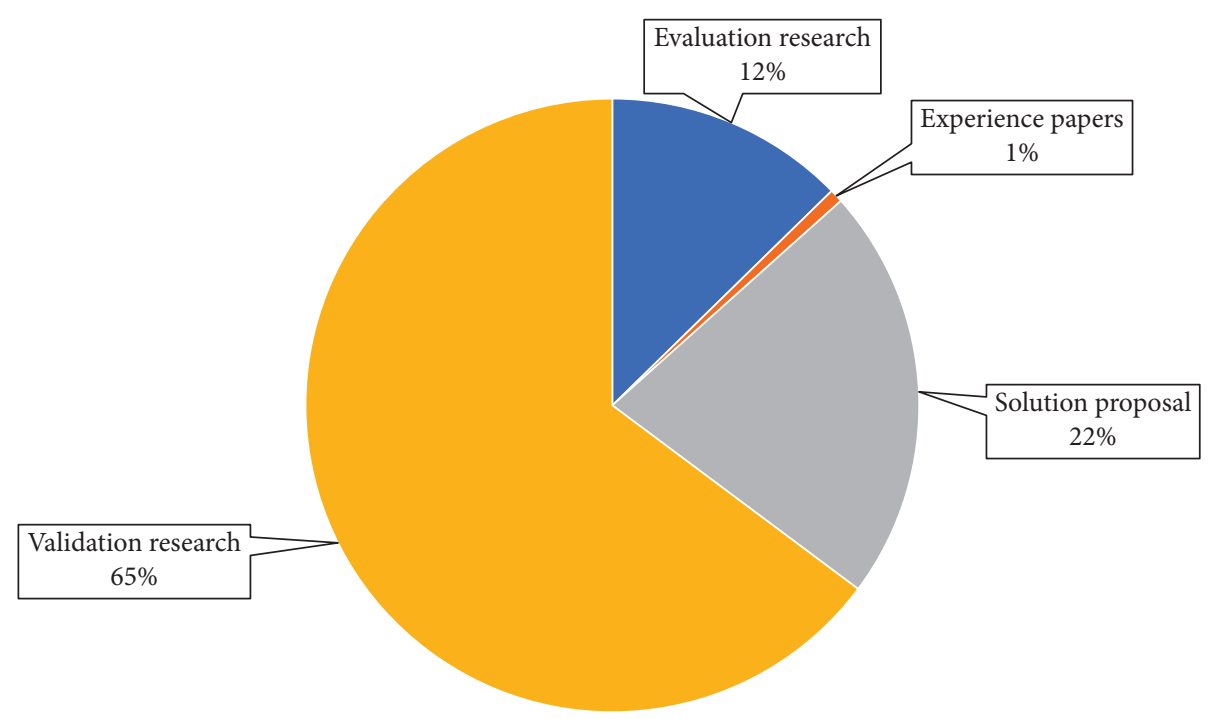

Figure 6: Research type classification.

the earliest deadline-harvesting (ED-H) scheduling algorithm, could save up to $33 \%$ capacity, while the remaining $37 \%$ was applied research. It is worth noting that energy management accounted for a large part of the papers. There has been little research into applications; however, since 2013 , the number of research papers concentrated on applications has been increasing. Hence, we believe that applications will become a main focus in this field and, so, energy management still has great potential.

Another important point is to determine the most appropriate method for each research goal, as shown in Figure 8. As the most popular type of research in the four categories was validation research, and as many validation research and solution proposal papers relate to applications, this indicates that the research type most suitable for energy management is validation research, followed by solution proposal research.

3.4. RQ3: What Are the Application Scenarios for the Energy Management of EHES? A total of 32\% (45 out of 142) of the 142 papers described application scenarios. As shown in Figure 9, wearable equipment accounted for 20\% (9 out of 45 ), medicine accounted for $11 \%$ (5 out of 45 ), buildings accounted for $36 \%$ (16 out of 45 ), and machines accounted for $33 \%$ (15 out of 45 ).

The theme of wearable equipment mainly included shoes, watches, glasses, and some equipment which pays attention to human health. These devices are powered by the vibrational energy or radiated heat generated by human activity. The theme of medicine is mainly concerned with devices implanted in the human body; for example, Murali et al. [10] proposed a solar energy harvesting power device for use with ocular implants. The theme of buildings mainly considers the monitoring of structural health or collection of information from bridges, buildings, and road, among others; for example, Jasim et al. [25] proposed energy harvesting from piezoelectric modules in asphalt pavement. The machine topic focuses on vehicles, airplanes, and so on, monitoring the health of parts of these machines or providing energy for certain systems in their parts which operate separately; for example, Yaqub and Heidary [38] proposed embedding a piezoelectric material inside of electric vehicle (EV) tyres to mathematically model harvested energy, as well as to collect and process mechanical stress data. The number of research papers in the medicine, building, and machine themes from 2005 to 2019 remained relatively stable, indicating that these three themes have not had much breakthrough research in recent years.

3.5. RQ4: How Many Tool Papers and Method Papers Were Proposed? From 2005 to 2019, a total of 12 tool papers and 81 method papers were published. Our focus was on finding tool papers and method papers which can help other researchers in this field in the future.

In the 12 tool papers found, their purpose was to run an embedded system for a long time and provide energy. Introduced in these papers, most notably, were Heliomote, a plug-and-play solar energy harvesting module [158], and a microscale energy harvesting simulation tool [112]. An important finding here is that most tool papers were designed for energy management or monitoring system status. This demonstrates the importance of health- and energy-related issues when the collection system is running. Of the 81 method papers found, the main purposes were (1) to manage the energy of the system itself, in order to ensure long-time operations and (2) to improve the conversion rate between renewable energy and electric energy. The most notable contributions of these papers included Hibernus [64], a new approach for sustaining computation under intermittent supply; a scheduling algorithm [105, 122, 125]; and dynamic voltage and frequency scaling [5]. An important finding here is that most method papers have been carried out through a large number of simulation experiments, and few algorithms have been implemented in practice. This demonstrates that algorithmic research may encounter some difficulties when attempting to translate 


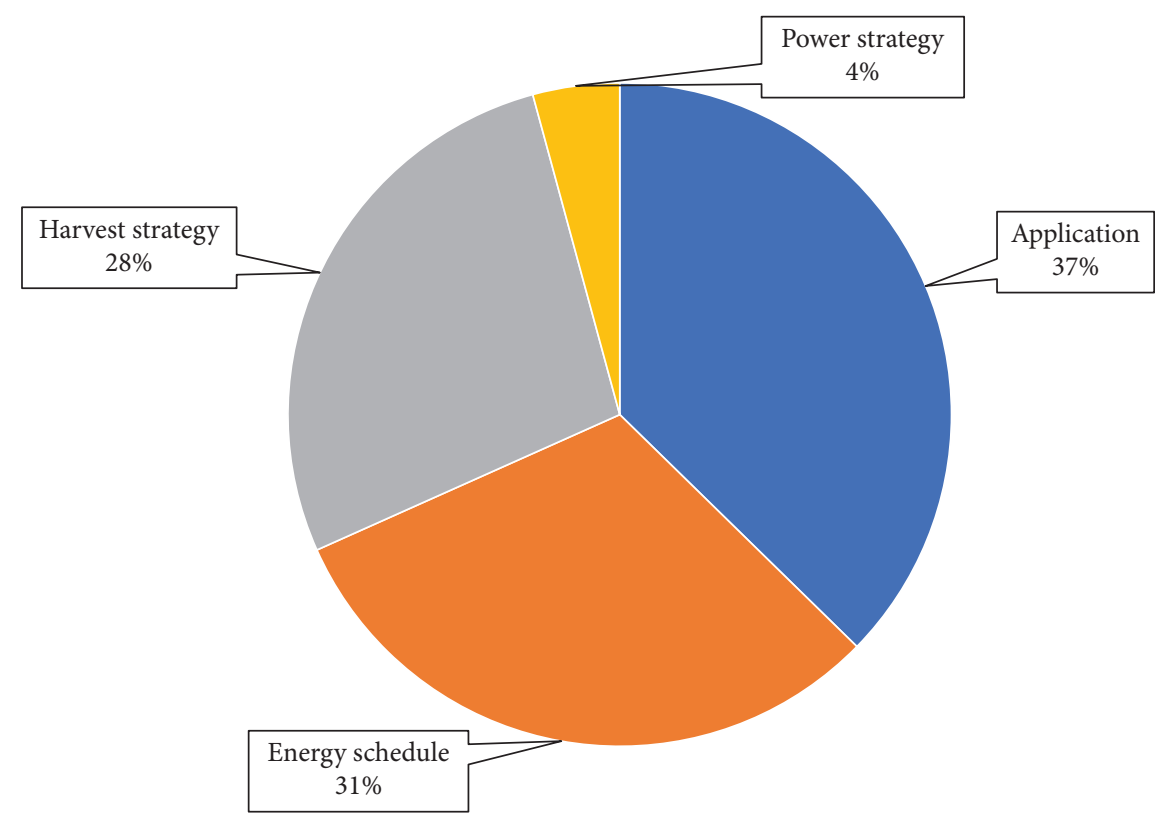

FIgURE 7: Research goals classification.

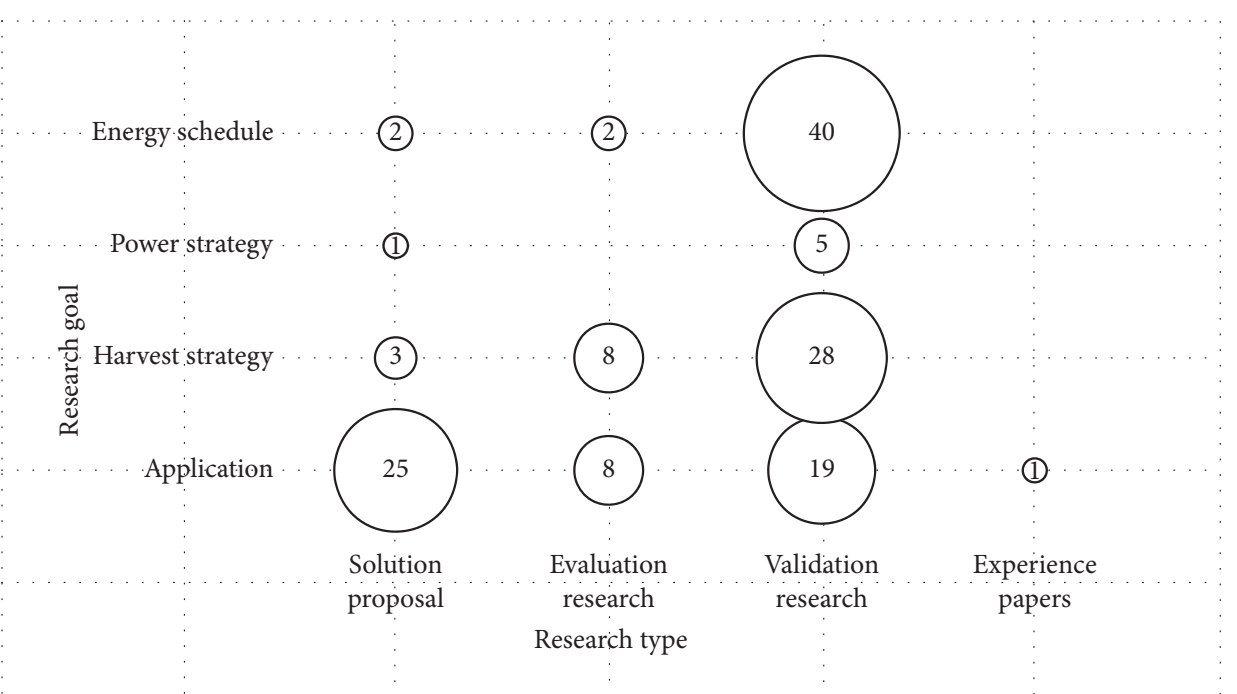

FIGURE 8: Research goals per research types.

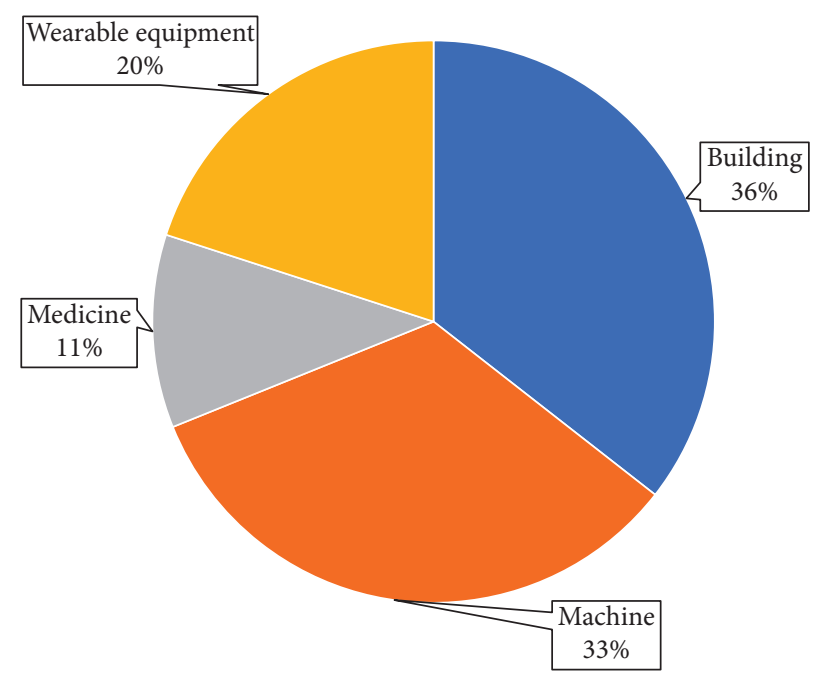

Figure 9: Application scenarios classification. 
into practice, which requires further research to be achieved. In the future, researchers need to publish more useful tools to facilitate the research of useful methods their application in practice.

\subsection{RQ5: How Are the Papers Distributed?}

3.6.1. Distribution by Publication Types. As shown in Figure $10,46 \%$ of authors (65 papers) selected conference publication, $42 \%$ (60 papers) selected journal publication, $8 \%$ (11 papers) selected symposium publication, and 3\% (5 papers) selected workshop publication. Therefore, there were more conference papers than journal papers, which indicates that the technology update in this domain is faster, and conferences can provide researchers with a platform for communication to promote their own research progress.

3.6.2. Distribution of Categories by Publishers. Figure 11 shows that EHES papers were published by different publishers. The review of this work consists of two categories (application and theory). The theory consisted of three ties as follows: energy schedule, harvest strategy, and power strategy.

ACM published 31 papers in different categories, which were composed of application (6), energy schedule (12), harvest strategy (11), and power strategy (2). IEEE Xplore published 67 papers, which were composed of application (24), energy schedule (24), harvest strategy (17), and power strategy (2). Spring published 6 papers, which were composed of application (3), energy schedule (1), harvest strategy (1), and power strategy (1). Wiley only published 1 paper, which is the application. Other publishers published 37 papers, which were composed of application (19), energy schedule (7), harvest strategy (10), and power strategy (1). About $74 \%$ of the papers were published by IEEE, ACM, Springer, and Wiley. This suggests that the research in this field has been recognized and appreciated by popular publishers, thus demonstrating that the field has great future potential.

3.6.3. Distribution by Publication Years. As shown in Figure 12, the number of publications per year from 2005 to 2019 gradually increased from 2005 to 2013. In particular, the number of publications in 2013 was almost three times that of 2007, 2008, and 2009. Although it declined in the three years after 2013, the number of publications then increased again from 2017. This work only selected papers before July 2019, and we believe that the number published in 2019 will exceed those in 2018, as the field has great potential based on recent trends.

3.6.4. Distribution by Nationality of the Author. Figure 13 shows the distribution of EHES research in 23 countries. We noticed that the research was conducted in these countries, or they covered cases in these countries.

The nationality distribution of the 142 EHES papers in numbers shows that the most productive authors are from the USA (44), followed by China (19); France (15); Italy (10); UK (8); Korea and India (5 each); Switzerland and Germany (4 each); Australia, Ireland, Japan, and Pakistan (3 each); Czech, Iraq, Norway, Lebanon, Portugal, and Spain (2 each); and Algeria, Montenegro, the Netherlands, and Turkey (each 1).

We believe that the above data can help new researchers relevant in this field to find suitable publishing venues to publish their future research.

\section{Challenges}

In this section, two future challenges will be encountered in the processes of EHES energy management.

4.1. Challenge of Energy Management. The most persistent and critical challenges in EHES are related to energy management, such as system scheduling, energy consumption, energy harvesting, and energy distribution. Compared with battery-powered embedded systems, the energy supply of EHES is unpredictable and unreliable. In this case, how to ensure the normal scheduling of system tasks is a signification problem. In this regard, many researchers have proposed various approaches, for example, global controller track the optimal operating point of the photovoltaic panel, state of charge management for the supercapacitor, and energy harvesting real-time task scheduling with DVFS in the embedded device [5]; the time constraints of battery-powered embedded devices are extended to energy constraints $[35,58,65,105,109]$; a new strategy was designed in conjunction with traditional battery-powered embedded system scheduling algorithm after considering energy attributes [62, 93, 97, 107]. Energy consumption has always been a long-term problem for embedded systems. Due to the power supply problem of the EHES system, the traditional battery-powered embedded system method cannot be used for EHES. Usually, researchers combine energy harvesting with traditional methods to form new methods $[75,161,162]$. Moreover, energy harvesting needs to consider how to stably and efficiently convert the collected energy [54, 129, 136, 137]. Lastly, how to reasonably supply power to embedded devices distributed in different geographical locations is a challenge currently facing energy distribution. At present, the main method is to use reinforcement learning for configuration $[23,36,45]$.

4.2. Challenge of Application. The application of EHES mainly faces the problem of safety. There are two main limiting factors, and they may cause several potential security risks. These problems are mainly caused by environmental factors. Firstly, the energy obtained from the environment is difficult to predict, that is, the amount of electricity generated is random, and when the harvested energy is converted into electric energy, there is a low conversion rate. Under such conditions, it may be difficult to provide equipment continuously sufficient power. If the EHES is transmitting data, data loss may occur. Secondly, 


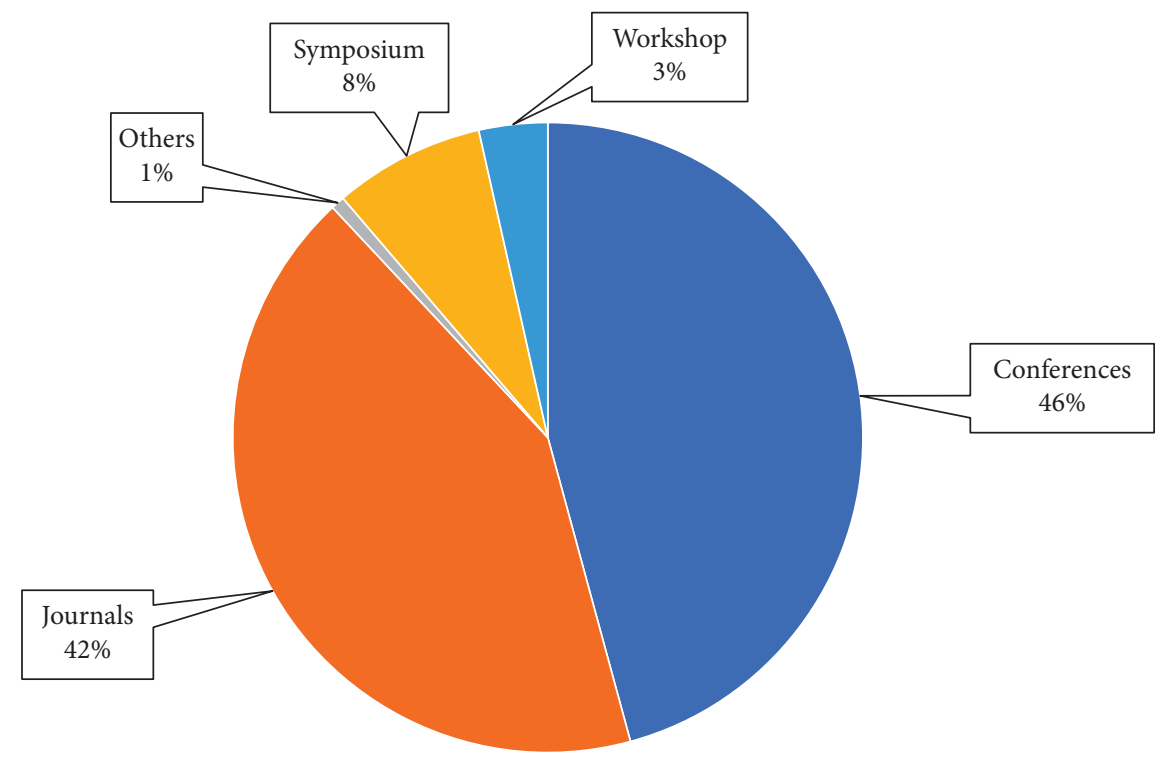

Figure 10: Publication types classification.

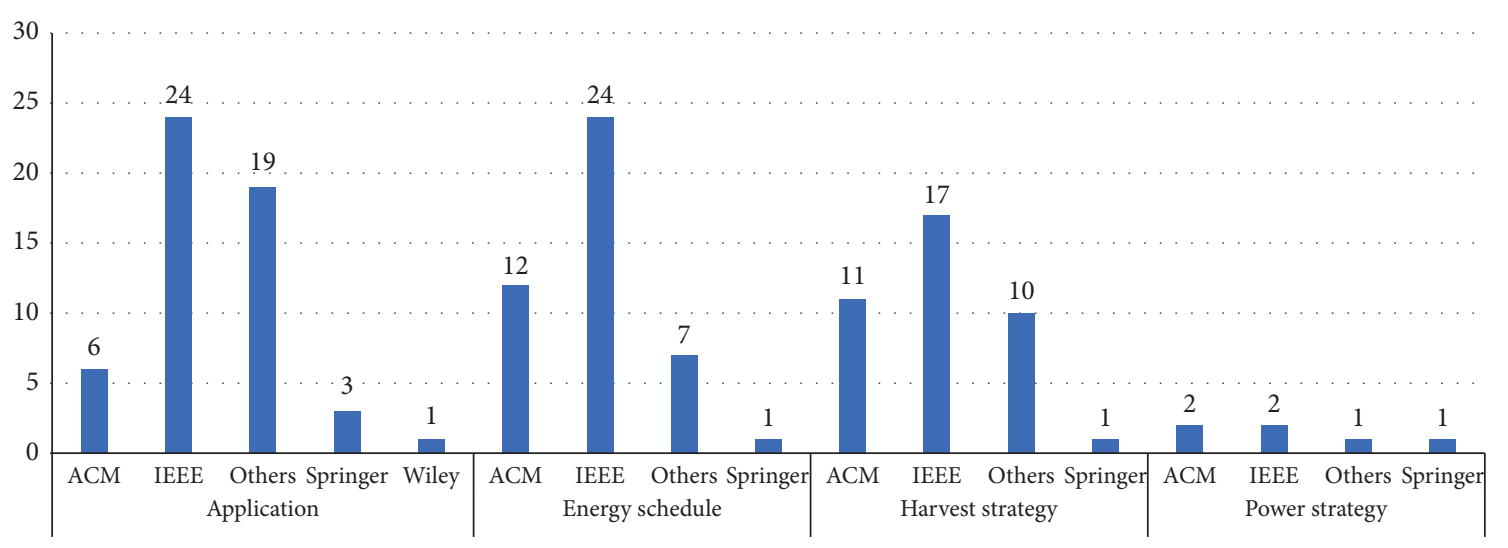

FIgURE 11: Statistics of the included papers in four different categories by publishers.

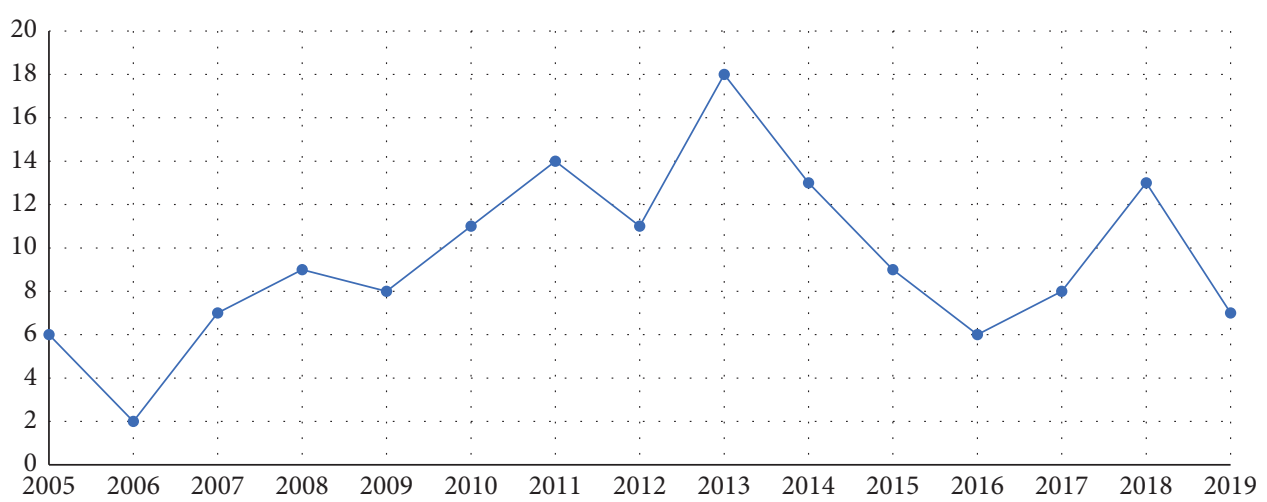

FIGURE 12: Statistics of the included papers by publication year.

due to differences in physical environment or harvested energy, for example, there are corrosive substances in the environment and energy, which can damage the hardware, the EHES will be damaged and will stop operating [163].
These limiting factors are potential sources of threats to EHES. When devices are powered by energy harvesting sources, they face other threats from attackers who can change the environment. For example, the RF source may be 


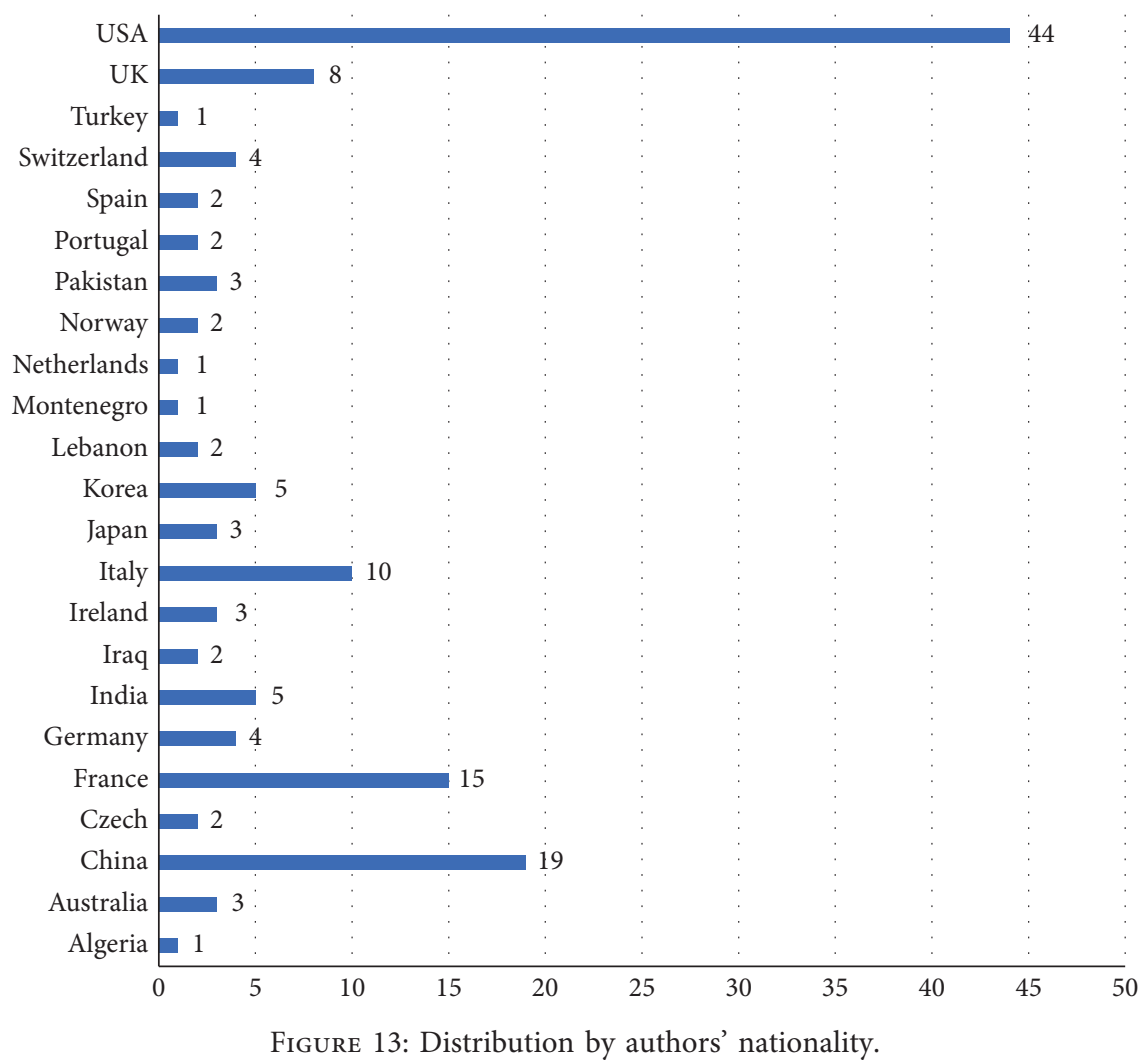

blocked and the device cannot send their data [164]. There have been researches on attacks and privacy [165-167]. However, the research is still in its infancy and lacks applicability, and many problems remain unsolved.

\section{Conclusion}

Battery-powered embedded devices have been widely used in various intelligent terminals. At the same time, intelligent terminals that adapt energy harvesting technology have attracted increasing attention. This type of intelligent terminals can solve the situation that intelligent terminals are difficult to charge or to replace batteries. This work contributes to extant published EHES energy management research by a systematic mapping study. We mainly classify papers by answering five questions and comprehensively analysed related papers by highlighting the number of publications, research types, research goals, application scenarios, tools, and methods. Some suggestions for EHES to help researchers find suitable research directions. Furthermore, we also analyse the challenges faced by EHES energy management and the possible implementation plans in the future. Because countries attach importance to sensitive information and privacy issues, researchers need to focus on the security of EHES in the future. At the same time, researchers must continue to deepen the existing research on energy management to ensure the sustainability of EHES.

Our future work will be devoted to the implementation of a systematic literature review (SLR) on the safety of EHES. In addition, we will continue to pay attention to the latest research on EHES energy management.

\section{Data Availability}

The data used to support the findings of this paper are included within the article.

\section{Conflicts of Interest}

The authors declare that they have no conflicts of interest.

\section{Acknowledgments}

This work was supported in part by the National Natural Science Foundation of China under Grant 61862049, in part by the Ningxia Key Research and Development Projects under Grant 2018BEB04020, and in part by the Young Scholar in Western China of Chinese Academy of Sciences under Grant XAB2018AW12.

\section{References}

[1] C. Lee, "Sensor as a solution: recent progress in intelligent sensors development," in Proceedings of the 2019 IEEE 32nd International Conference on Micro Electro Mechanical Systems (MEMS), p. 256, IEEE, Seoul, Republic of Korea, 2019.

[2] J. Wang, Y. Chen, S. Hao, X. Peng, and L. Hu, "Deep learning for sensor-based activity recognition: a survey," Pattern Recognition Letters, vol. 119, pp. 3-11, 2019.

[3] X. Chen, Z. Xu, H. Kim et al., "Dynamic voltage and frequency scaling for shared resources in multicore processor designs," in Proceedings of the 2013 50th ACM/EDAC/IEEE Design Automation Conference (DAC), Austin, TX, USA, 2013. 
[4] S. Saha and B. Ravindran, "An experimental evaluation of real-time DVFS scheduling algorithms," in Proceedings of the 5th Annual International Conference on Systems and Storage-SYSTOR'12, pp. 1-12, Haifa, Israel, 2012.

[5] X. Lin, Y. Wang, S. Yue, N. Chang, and M. Pedram, "A framework of concurrent task scheduling and dynamic voltage and frequency scaling in real-time embedded systems with energy harvesting," in Proceedings of the 2013 International Symposium on Low Power Electronics and Design (ISLPED), Beijing, China, 2013.

[6] K. Huang, L. Santinelli, J.-J. Chen, L. Thiele, and G. C. Buttazzo, "Applying real-time interface and calculus for dynamic power management in hard real-time systems," Real-Time Systems, vol. 47, no. 2, pp. 163-193, 2011.

[7] W. Dargie, "Dynamic power management in wireless sensor networks: state-of-the-art," IEEE Sensors Journal, vol. 12, no. 5, pp. 1518-1528, 2012.

[8] Q. Qiu, S. Liu, and Q. Wu, “Task merging for dynamic power management of cyclic applications in real-time multiprocessor systems," in Proceedings of the 2006 International Conference on Computer Design, San Jose, CA, USA, 2006.

[9] M. P. Soares dos Santos, J. A. F. Ferreira, A. Ramos et al., "Instrumented hip implants: electric supply systems," Journal of Biomechanics, vol. 46, no. 15, pp. 2561-2571, 2013.

[10] K. Murali, N. Scianmarello, and M. S. Humayun, "Harvesting solar energy to power ocular implants," in Proceedings of the 2015 IEEE Biomedical Circuits and Systems Conference (BioCAS), Atlanta, GA, USA, 2015.

[11] S. K. S. Gupta, T. Mukherjee, G. Varsamopoulos, and A. Banerjee, "Research directions in energy-sustainable cyber-physical systems," Sustainable Computing: Informatics and Systems, vol. 1, no. 1, pp. 57-74, 2011.

[12] S. Keele, "Guidelines for performing systematic literature reviews in software engineering," Technical report, Keele University, Newcastle, UK, 2007.

[13] K. Petersen, R. Feldt, S. Mujtaba, and M. Mattsson, "Systematic mapping studies in software engineering," in Proceedings of the 12th International Conference on Evaluation and Assessment in Software Engineering (EASE), Bari, Italy, 2008.

[14] K. Petersen, S. Vakkalanka, and L. Kuzniarz, "Guidelines for conducting systematic mapping studies in software engineering: an update," Information and Software Technology, vol. 64, pp. 1-18, 2015.

[15] B. Kitchenham, O. Pearl Brereton, D. Budgen, M. Turner, J. Bailey, and S. Linkman, "Systematic literature reviews in software engineering-a systematic literature review," Information and Software Technology, vol. 51, no. 1, pp. 7-15, 2009.

[16] A. Kofod-Petersen, "How to do a structured literature review in computer science," 2012, https://research.idi.ntnu.no/ aimasters/files/SLR_HowTo2018.pdf.

[17] B. Kitchenham, Procedures for Performing Systematic Reviews, Keele University, Keele, UK, 2004.

[18] C. Wohlin, "Guidelines for snowballing in systematic literature studies and a replication in software engineering," in Proceedings of the 18th International Conference on Evaluation and Assessment in Software Engineering-EASE '14, London, UK, 2014.

[19] R. Wieringa, N. Maiden, N. Mead, and C. Rolland, "Requirements engineering paper classification and evaluation criteria: a proposal and a discussion," Requirements Engineering, vol. 11, no. 1, pp. 102-107, 2006.
[20] M. E. Yüksel, "Design and implementation of A batteryless wireless embedded system for IoT applications," Electrica, vol. 19, no. 1, pp. 1-11, 2019.

[21] H. Xu, R. Li, L. Zeng, K. Li, and C. Pan, "Energy-efficient scheduling with reliability guarantee in embedded real-time systems," Sustainable Computing: Informatics and Systems, vol. 18, pp. 137-148, 2018.

[22] M. Shirvanimoghaddam, K. Shirvanimoghaddam, M. M. Abolhasani et al., "Towards a green and self-powered Internet of things using piezoelectric energy harvesting," IEEE Access, vol. 7, pp. 94533-94556, 2019.

[23] Y. Xu, H. G. Lee, Y. Tan et al., "Tumbler: energy efficient task scheduling for dual-channel solar-powered sensor nodes," in Proceedings of the 2019 56th ACM/IEEE Design Automation Conference (DAC), Las Vegas, NV, USA, 2019.

[24] R. Ahmed, B. Buchli, S. Draskovic, L. Sigrist, P. Kumar, and L. Thiele, "Optimal power management with guaranteed minimum energy utilization for solar energy harvesting systems," ACM Transactions on Embedded Computing Systems, vol. 18, no. 4, pp. 1-26, 2019.

[25] A. F. Jasim, H. Wang, G. Yesner, A. Safari, and P. Szary, "Performance analysis of piezoelectric energy harvesting in pavement: laboratory testing and field simulation," Transportation Research Record: Journal of the Transportation Research Board, vol. 2673, no. 3, , 2019, https://journals. sagepub.com/doi/abs/10.1177/0361198119830308.

[26] R. Esmaeeli, H. Aliniagerdroudbari, S. R. Hashemi et al., "A rainbow piezoelectric energy harvesting system for intelligent tire monitoring applications," Journal of Energy Resources Technology, vol. 141, no. 6, Article ID 062007, 2019.

[27] F. U. Khan and T. Ali, "A piezoelectric based energy harvester for simultaneous energy generation and vibration isolation," International Journal of Energy Research, vol. 43, no. 11, pp. 5922-5931, 2019.

[28] R. A. Kjellby, L. R. Cenkeramaddi, T. E. Johnsrud et al., "Selfpowered IoT device based on energy harvesting for remote applications," in Proceedings of the 2018 IEEE International Conference on Advanced Networks and Telecommunications Systems (ANTS), Indore, India, 2018.

[29] F. Fraternali, B. Balaji, and R. Gupta, "Scaling configuration of energy harvesting sensors with reinforcement learning," in Proceedings of the 6th International Workshop on Energy Harvesting \& Energy-Neutral Sensing Systems-ENSsys 18, Shenzhen, China, 2018.

[30] A. Colin, E. Ruppel, and B. Lucia, "A reconfigurable energy storage architecture for energy-harvesting devices," in Proceedings of the 23rd International Conference on Architectural Support for Programming Languages and Operating Systems, pp. 767-781, ACM, Williamsburg, VA, USA, 2018.

[31] J.-M. Gruber and A. Stahel, "Self-powered sensor with energy harvesting from human walking," in Proceedings of the 19th ITG/GMA-Symposium on Sensors and Measuring Systems, Nuremberg, Germany, 2018.

[32] G. Laštovička-Medin, "Harvesting regenerating energy from multiple low-cost energy generators for low energy consumers and towards circular economy," in Proceedings of the 2018 7th Mediterranean Conference on Embedded Computing (MECO), Budva, Montenegro, 2018.

[33] X. Liu, L. Yu, S. Zheng, and J. Chang, "Energy harvesting in tire: state-of-the-art and challenges," SAE International Journal of Passenger Cars-Electronic \& Electrical Systems, vol. 11, pp. 159-170, 2018.

[34] A. M. Nair, V. Surya, R. Apoorva, K. G. Singh, K. Chandan, and B. H. Nidha, "Harvesting energy from pavements," in 
Proceedings of GeoShanghai 2018 International Conference: Transportation Geotechnics and Pavement Engineering, pp. 408-415, Springer, Singapore, 2018.

[35] R. E. Osta, M. Chetto, and H. E. Ghor, "An optimal approach for minimizing aperiodic response times in real-time energy harvesting systems," in Proceedings of the 2018 IEEE/ACS 15th International Conference on Computer Systems and Applications (AICCSA), Aqaba, Jordan, 2018.

[36] M. Prauzek, N. R. A. Mourcet, J. Hlavica, and P. Musilek, "Qlearning algorithm for energy management in solar powered embedded monitoring systems," in Proceedings of the 2018 IEEE Congress on Evolutionary Computation (CEC), Rio de Janeiro, Brazil, 2018.

[37] R. A. Kjellby, T. E. Johnsrud, S. E. Loetveit, L. R. Cenkeramaddi, M. Hamid, and B. Beferull-Lozano, "Self-powered IoT device for indoor applications," in Proceedings of the 2018 31st International Conference on VLSI Design and 2018 17th International Conference on Embedded Systems (VLSID), Pune, India, 2018.

[38] R. Yaqub and K. Heidary, "Mathematical modelling of energy harvesting in piezo embedded electric vehicle tyres together with self-health assessment of suspension system," International Journal of Electric and Hybrid Vehicles, vol. 10, no. 2, p. 161, 2018.

[39] M. Prauzek, J. Konecny, M. Borova, K. Janosova, J. Hlavica, and P. Musilek, "Energy harvesting sources, storage devices and system topologies for environmental wireless sensor networks: a review," Sensors, vol. 18, no. 8, p. 2446, 2018.

[40] G. Bhat, J. Park, and U. Y. Ogras, "Near-optimal energy allocation for self-powered wearable systems," in Proceedings of the 2017 IEEE/ACM International Conference on Computer-Aided Design (ICCAD), Irvine, CA, USA, 2017.

[41] Y. Deng, L. Liao, Y. Wu et al., "Self-powered intelligent door handle based on triboelectric nanogenerator," in Proceedings of the 2017 IEEE 16th International Conference on Cognitive Informatics \& Cognitive Computing, Oxford, UK, 2017.

[42] J. Heo and M. Park, "Simulation analysis of power management techniques for a solar-powered embedded device," in Proceedings of the 2017 2nd International Conference on Power and Renewable Energy (ICPRE), Chengdu, China, 2017.

[43] X. Li, N. Xie, and X. Tian, "Dynamic voltage-frequency and workload joint scaling power management for energy harvesting multi-core WSN node SoC," Sensors, vol. 17, no. 2, p. 310, 2017.

[44] C. Pan, M. Xie, and J. Hu, "Maximize energy utilization for ultra-low energy harvesting powered embedded systems," in Proceedings of the 2017 IEEE 23rd International Conference on Embedded and Real-Time Computing Systems and Applications (RTCSA), Hsinchu, Taiwan, 2017.

[45] S. Shresthamali, M. Kondo, and H. Nakamura, "Adaptive power management in solar energy harvesting sensor node using reinforcement learning," ACM Transactions on Embedded Computing Systems, vol. 16, no. 5, pp. 1-21, 2017.

[46] Y. Zhou, M. Zhao, L. Ju, C. J. Xue, X. Li, and Z. Jia, "Energyaware morphable cache management for self-powered nonvolatile processors," in Proceedings of the 2017 IEEE 23rd International Conference on Embedded and Real-Time Computing Systems and Applications (RTCSA), Hsinchu, Taiwan, 2017.

[47] C. Wei and X. Jing, "A comprehensive review on vibration energy harvesting: modelling and realization," Renewable and Sustainable Energy Reviews, vol. 74, pp. 1-18, 2017.
[48] D. Brunelli, "A high-efficiency wind energy harvester for autonomous embedded systems," Sensors, vol. 16, no. 3, p. 327, 2016.

[49] A. C. Galbier and M. A. Karami, "A bistable piezoelectric energy harvester with an elastic magnifier for applications in medical pacemakers," Volume 2: Modeling, Simulation and Control; Bio-Inspired Smart Materials and Systems; Energy Harvesting, American Society of Mechanical Engineers (ASME), Stowe, VT, USA, 2016.

[50] Q. Ibrahim, "Enhanced power management scheme for embedded road side units," IET Computers \& Digital Techniques, vol. 10, no. 4, pp. 174-185, 2016.

[51] S. Mondal and R. P. Paily, "An efficient on chip power management architecture for solar energy harvesting systems," in Proceedings of the 2016 29th International Conference on VLSI Design and 2016 15th International Conference on Embedded Systems (VLSID), Kolkata, India, 2016.

[52] Y. Tan and X. Yin, "A dynamic scheduling algorithm for energy harvesting embedded systems," EURASIP Journal on Wireless Communications and Networking, vol. 2016, no. 1, p. 114, 2016.

[53] T. Zou, S. Lin, Q. Feng, and Y. Chen, "Energy-efficient control with harvesting predictions for solar-powered wireless sensor networks," Sensors, vol. 16, no. 1, p. 53, 2016.

[54] K. Geissdoerfer, R. Jurdak, B. Kusy, and M. Zimmerling, "Getting more out of energy-harvesting systems: energy management under time-varying utility with P re A ct," in Proceedings of the 18th International Conference on Information Processing in Sensor Networks, Montreal, Canada, 2019.

[55] B. Buchli, P. Kumar, and L. Thiele, "Optimal power management with guaranteed minimum energy utilization for solar energy harvesting systems," in Proceedings of the 2015 International Conference on Distributed Computing in Sensor Systems, Fortaleza, Brazil, 2015.

[56] N. Dang, R. Valentini, E. Bozorgzadeh, M. Levorato, and N. Venkatasubramanian, "A unified stochastic model for energy management in solar-powered embedded systems," in Proceedings of the 2015 IEEE/ACM International Conference on Computer-Aided Design (ICCAD), Austin, TX, USA, 2015.

[57] S. Heidari, C. Ding, Y. Liu, Y. Wang, and J. Hu, "Multisource energy harvesting management and optimization for non-volatile processors," in Proceedings of the 2015 6th International Green and Sustainable Computing Conference (IGSC), Las Vegas, NV, USA, 2015.

[58] H. E. Ghor and M. Chetto, "Overhead considerations in realtime energy harvesting systems," in Proceedings of the 2015 International Conference on Pervasive and Embedded Computing and Communication Systems (PECCS), Angers, France, 2015.

[59] V. S. Rao, R. V. Prasad, and I. G. M. M. Niemegeers, "Optimal task scheduling policy in energy harvesting wireless sensor networks," in Proceedings of the 2015 IEEE Wireless Communications and Networking Conference (WCNC), Shanghai, China, 2015.

[60] Y. Xiang and S. Pasricha, "Run-time management for multicore embedded systems with energy harvesting," IEEE Transactions on Very Large Scale Integration (VLSI) Systems, vol. 23, no. 12, pp. 2876-2889, 2015.

[61] L. Xie and M. Cai, "An in-shoe harvester with motion magnification for scavenging energy from human foot 
strike," IEEE/ASME Transactions on Mechatronics, vol. 20, no. 6, pp. 3264-3268, 2015.

[62] Y. Abdeddaïm, Y. Chandarli, R. I. Davis, and D. Masson, "Schedulability analysis for fixed priority real-time systems with energy-harvesting," in Proceedings of the 22nd International Conference on Real-Time Networks and Systems, pp. 311-320, ACM Press, Versaille, France, 2014.

[63] K. Aono, A. Iwata, H. Takase, K. Takagi, and N. Takagi, "An operation scenario model for energy harvesting embedded systems and an algorithm to maximize the operation quality," in Proceedings of the 2014 IEEE International Conference on High Performance Computing and Communications, 2014 IEEE 6th International Symposium on Cyberspace Safety and Security, 2014 IEEE 11th International Conference on Embedded Software and Syst (HPCC, CSS, ICESS), Paris, France, 2014.

[64] D. Balsamo, A. S. Weddell, G. V. Merrett, B. M. Al-Hashimi, D. Brunelli, and L. Benini, "Hibernus: sustaining computation during intermittent supply for energy-harvesting systems," IEEE Embedded Systems Letters, vol. 7, no. 1, pp. 15-18, 2015.

[65] M. Chetto and A. Queudet, "A note on EDF scheduling for real-time energy harvesting systems," IEEE Transactions on Computers, vol. 63, no. 4, pp. 1037-1040, 2014.

[66] A. Gatto and E. Frontoni, "Energy Harvesting system for smart shoes," in Proceedings of the 2014 IEEE/ASME 10th International Conference on Mechatronic and Embedded Systems and Applications (MESA), Senigallia, Italy, 2014.

[67] T. N. Le, A. Pegatoquet, O. Berder, and O. Sentieys, "A power manager with balanced quality of service for energy-harvesting wireless sensor nodes," in Proceedings of the 2nd International Workshop on Energy Neutral Sensing Systems, pp. 19-24, ACM Press, Memphis, Tennessee, 2014.

[68] Q. Liu, T. Mak, T. Zhang, X. Niu, W. Luk, and A. Yakovlev, "Power-adaptive computing system design for solar-energypowered embedded systems," IEEE Transactions on Very Large Scale Integration (VLSI) Systems, vol. 23, no. 8, pp. 1402-1414, 2015.

[69] D. Milani, M. Bassetti, F. Braghin, and G. Tomasini, "Design of a wireless sensor powered by a piezoelectric energy harvester," Volume 3: Engineering Systems; Heat Transfer and Thermal Engineering; Materials and Tribology; Mechatronics; Robotics, American Society of Mechanical Engineers (ASME), Copenhagen, Denmark, 2014.

[70] H. Patsamatla, V. Karthikeyan, and R. Gupta, "Universal maximum power point tracking in wind-solar hybrid system for battery storage application," in Proceedings of the 2014 International Conference on Embedded Systems (ICES), Las Vegas, NV, USA, 2014.

[71] Y. Xiang and S. Pasricha, "A hybrid framework for application allocation and scheduling in multicore systems with energy harvesting," in Proceedings of the 24th edition of the great lakes symposium on VLSI, pp. 163-168, ACM Press, Houston, TX, USA, 2014.

[72] J. Zhao and Z. You, "A shoe-embedded piezoelectric energy harvester for wearable sensors," Sensors, vol. 14, no. 7, pp. 12497-12510, 2014.

[73] H. Zhao, Y. Tao, Y. Niu, and J. Ling, "Harvesting energy from asphalt pavement by piezoelectric generator," Journal of Wuhan University of Technology-Materials Science Edition, vol. 29, no. 5, pp. 933-937, 2014.

[74] D. Gunduz, K. Stamatiou, N. Michelusi, and M. Zorzi, "Designing intelligent energy harvesting communication systems," IEEE Communications Magazine, vol. 52, no. 1, pp. 210-216, 2014.

[75] A. Abbas, E. Grolleau, M. Loudini, and D. Mehdi, “A realtime feedback scheduler for environmental energy harvesting," in Proceedings of the 3rd International Conference on Systems and Control, Algiers, Algeria, 2013.

[76] S. R. Anton, S. G. Taylor, E. Y. Raby, and K. M. Farinholt, "Powering embedded electronics for wind turbine monitoring using multi-source energy harvesting techniques," in Proceedings of the 2013 SPIE Smart Structures and Materials + Nondestructive Evaluation and Health Monitoring, San Diego, CA, USA, 2013.

[77] G. Huang, R. Umaz, U. Karra, B. Li, and L. Wang, "A biomass-based marine sediment energy harvesting system," in Proceedings of the 2013 International Symposium on Low Power Electronics and Design (ISLPED), Beijing, China, 2013.

[78] A. Cammarano, D. Spenza, and C. Petrioli, "Energy-harvesting WSNs for structural health monitoring of underground train tunnels," in Proceedings of the 2013 IEEE Conference on Computer Communications Workshops, Turin, Italy, 2013.

[79] M. Choi, K. M. Farinholt, S. Anton, J.-R. Lee, and G. Park, "Multi-source energy harvesting for wireless SHM systems," in Proceedings of the 2013 SPIE Smart Structures and Materials + Nondestructive Evaluation and Health Monitoring, San Diego, CA, USA, 2013.

[80] K. Lee and T. Ishihara, "DC-DC converter-aware task scheduling and dynamic reconfiguration for energy harvesting embedded systems," IEICE Transactions on Fundamentals of Electronics, Communications and Computer Sciences, vol. E96.A, no. 12, pp. 2660-2667, 2013.

[81] Q. Ibrahim, "Design, implementation and optimisation of an energy harvesting system for vehicular ad hoc networks' road side units," IET Intelligent Transport Systems, vol. 8, no. 3, pp. 298-307, 2014.

[82] X. Jiang, Y. Li, and J. Li, "A piezoelectric wafer-stack vibration energy harvester for wireless sensor networks," in Proceeedings of the 2013 SPIE Smart Structures and Materials + Nondestructive Evaluation and Health Monitoring, San Diego, CA, USA, 2013.

[83] K. H. Mak, S. McWilliam, and A. A. Popov, "Piezoelectric energy harvesting for tyre pressure measurement applications," Proceedings of the Institution of Mechanical Engineers, Part D: Journal of Automobile Engineering, vol. 227, no. 6, pp. 842-852, 2013.

[84] C. Mo and J. Davidson, "Energy harvesting technologies for structural health monitoring applications," in Proceeedings of the 2013 1st IEEE Conference on Technologies for Sustainability, Portland, OR, USA, 2013.

[85] D. Porcarelli, D. Brunelli, and L. Benini, "Improving the efficiency of air-flow energy harvesters combining active and passive rectifiers," in Proceedings of the 1st International Workshop on Energy Neutral Sensing Systems, pp. 1-6, ACM Press, Rome, Italy, 2013.

[86] N. M. Silva, P. M. Santos, J. A. F. Ferreira et al., "Power management architecture for smart hip prostheses comprising multiple energy harvesting systems," Sensors and Actuators A: Physical, vol. 202, pp. 183-192, 2013.

[87] P. Sommer, B. Kusy, and R. Jurdak, "Power management for long-term sensing applications with energy harvesting," in Proceedings of the 1st International Workshop on Energy Neutral Sensing Systems, pp. 1-6, ACM Press, Rome, Italy, 2013. 
[88] A. S. Weddell, M. Magno, G. V. Merrett, D. Brunelli, B. M. Al-Hashimi, and L. Benini, "A survey of multi-source energy harvesting systems," in Proceedings of the 2013 Design, Automation Test in Europe Conference Exhibition (DATE), Grenoble, France, 2013.

[89] Ge Yang, Y. Zhang, and Q. Qiu, "Improving energy efficiency for energy harvesting embedded systems," in Proceedings of the 2013 18th Asia and South Pacific Design Automation Conference (ASP-DAC), Yokohama, Japan, 2013.

[90] H. D. Zhao, J. M. Ling, and P. C. Fu, "A review of harvesting green energy from road," Advanced Materials Research, vol. 723, pp. 559-566, 2013.

[91] L. Huang and M. J. Neely, "Utility optimal scheduling in energy-harvesting networks," IEEE/ACM Transactions on Networking, vol. 21, no. 4, pp. 1117-1130, 2013.

[92] A. Pang and R. Bannatyne, "The energy harvesting tipping point for wireless sensor applications," in Instrumentation, Measurement, Circuits and Systems, pp. 387-391, Springer, Berlin, Germany, 2012.

[93] M. Abdallah, M. Chetto, and A. Queudet, "Energy-aware schedulers for real-time energy harvesting systems with quality of service requirements," in Proceedings of the 2012 2nd International Conference on Advances in Computational Tools for Engineering Applications (ACTEA), Beirut, Lebanon, 2012.

[94] M. Abdallah, M. Chetto, and A. Queudet, "Scheduling with quality of service requirements in real-time energy harvesting sensors," in Proceedings of the 2012 IEEE International Conference on Green Computing and Communications, Besancon, France, 2012.

[95] P. Anacleto, P. M. Mendes, E. Gultepe, and D. H. Gracias, "3D small antenna for energy harvesting applications on implantable micro-devices," in Proceedings of the 2012 Loughborough Antennas Propagation Conference (LAPC), Loughborough, UK, 2012.

[96] M. Arnold, C. A. Featherston, M. R. Pearson, J. Lees, and A. Kural, "Energy management systems for energy harvesting in structural health monitoring applications," Key Engineering Materials, vol. 518, pp. 137-153, 2012.

[97] Y. Chandarli, Y. Abdeddaim, and D. Masson, "The fixed priority scheduling problem for energy harvesting real-time systems," in Proceedings of the 2012 IEEE International Conference on Embedded and Real-Time Computing Systems and Applications, pp. 415-418, IEEE, Seoul, Republic of Korea, 2012.

[98] J. F. Christmann, E. Beigné, C. Condemine, J. Willemin, and C. Piguet, "Energy harvesting and power management for autonomous sensor nodes," in Proceedings of the 49th Conference on Annual Design Automation, p. 1049, ACM Press, San Francisco, CA, USA, 2012.

[99] S.-G. Kim, S. Priya, and I. Kanno, "Piezoelectric MEMS for energy harvesting," MRS Bulletin, vol. 37, 2012.

[100] N. Kong and D. S. Ha, "Low-power design of a self-powered piezoelectric energy harvesting system with maximum power point tracking," IEEE Transactions on Power Electronics, vol. 27, no. 5, pp. 2298-2308, 2012.

[101] M. Kroener, "Energy harvesting technologies: energy sources, generators and management for wireless autonomous applications," in Proceedings of the 2012 International MultiConference on Systems, Signals Devices, Chemnitz, Germany, 2012.

[102] S. Liu, J. Lu, Q. Wu, and Q. Qiu, "Harvesting-aware power management for real-time systems with renewable energy,"
IEEE Transactions on Very Large Scale Integration VLSI Systems, vol. 20, no. 8, pp. 1473-1486, 2012.

[103] M. Abdallah, M. Chetto, A. Queudet, and R. H. Chehade, "Quality of service facilities for firm real-time energy harvesting systems," in Proceedings of the 2011 18th IEEE International Conference on Electronics, Circuits, and Systems, Beirut, Lebanon, 2011.

[104] E. Arroyo and A. Badel, "Electromagnetic vibration energy harvesting device optimization by synchronous energy extraction," Sensors and Actuators A: Physical, vol. 171, no. 2, pp. 266-273, 2011.

[105] M. Chetto, H. El Ghor, and R. H. Chehade, "Real-time scheduling for energy harvesting sensors," in Proceedings of the 2011 International Conference for Internet Technology and Secured Transactions, Abu Dhabi, UAE, 2011.

[106] D. Carroll and M. Duffy, "Modelling, design, and testing of an electromagnetic power generator optimized for integration into shoes," Proceedings of the Institution of Mechanical Engineers, Part I: Journal of Systems and Control Engineering, vol. 226, no. 2, pp. 256-270, 2012.

[107] M. Chetto, D. Masson, and S. Midonnet, "Fixed priority scheduling strategies for ambient energy-harvesting embedded systems," in Proceedings of the 2011 IEEE/ACM International Conference on Green Computing and Communications, Chengdu, China, 2011.

[108] J. Jessen, M. Venzke, and V. Turau, "Design considerations for a universal smart energy module for energy harvesting in wireless sensor networks," in Proceedings of the 2011 9th International Workshop on Intelligent Solutions in Embedded Systems, Regensburg, Germany, 2011.

[109] H. EL Ghor, M. Chetto, and R. H. Chehade, "A real-time scheduling framework for embedded systems with environmental energy harvesting," Computers \& Electrical Engineering, vol. 37, no. 4, pp. 498-510, 2011.

[110] Q. Liu, T. Mak, J. Luo, W. Luk, and A. Yakovlev, "Power adaptive computing system design in energy harvesting environment," in Proceedings of the Modeling and Simulation 2011 International Conference on Embedded Computer Systems: Architectures, Samos, Greece, 2011.

[111] J. Lu and Q. Qiu, "Scheduling and mapping of periodic tasks on multi-core embedded systems with energy harvesting," in Proceedings of the 2011 International Green Computing Conference and Workshops, Orlando, FL, USA, 2011.

[112] C. Lu, V. Raghunathan, and K. Roy, "Efficient design of micro-scale energy harvesting systems," IEEE Journal on Emerging and Selected Topics in Circuits and Systems, vol. 1, no. 3, pp. 254-266, 2011.

[113] Springer, Energy Management for Energy Harvesting Real Time System with Dynamic Voltage Scaling, SpringerLink, Berlin, Germany, 2011, https://link.springer.com/chapter/10. 1007/978-3-642-22543-7_55.

[114] H. Wang and L. Zou, "Parametric investigation of a piezoelectric energy harvester with elastic support," in Proceedings of the 2011 Symposium on Piezoelectricity, Acoustic Waves and Device Applications (SPAWDA), Shenzhen, China, 2011.

[115] M. Wischke, M. Masur, M. Kröner, and P. Woias, "Vibration harvesting in traffic tunnels to power wireless sensor nodes," Smart Materials and Structures, vol. 20, no. 8, Article ID 085014, 2011.

[116] H. S. Kim, J.-H. Kim, and J. Kim, "A review of piezoelectric energy harvesting based on vibration," International Journal of Precision Engineering and Manufacturing, vol. 12, no. 6, pp. 1129-1141, 2011. 
[117] M. I. Ali, B. M. Al-Hashimi, J. Recas, and D. Atienza, "Evaluation and design exploration of solar harvested-energy prediction algorithm," in Proceedings of the 2010 Design, Automation Test in Europe Conference Exhibition (DATE 2010), Dresden, Germany, 2010.

[118] K. M. Farinholt, S. G. Taylor, G. Park, and C. R. Farrar, "Wireless energy transmission to supplement energy harvesters in sensor network applications," in Proceedings of the SPIE Smart Structures and Materials + Nondestructive Evaluation and Health Monitoring, San Diego, CA, USA, 2010.

[119] K. M. Farinholt, N. Miller, W. Sifuentes, J. MacDonald, G. Park, and C. R. Farrar, "Energy harvesting and wireless energy transmission for embedded SHM sensor nodes," Structural Health Monitoring: An International Journal, vol. 9, no. 3, pp. 269-280, 2010.

[120] H.-W. Lee, J.-S. Kim, and B.-K. Lee, "Photovoltaic solar cell application for e-book," in Proceedings of the 2010 International Conference on Electrical Machines and Systems, Incheon, Republic of Korea, 2010.

[121] J. Lu, S. Liu, Q. Wu, and Q. Qiu, "Accurate modeling and prediction of energy availability in energy harvesting realtime embedded systems," in Proceedings of the 2010 International Conference on Green Computing, Chicago, IL, USA, 2010.

[122] S. Liu, J. Lu, Q. Wu, and Q. Qiu, "Load-matching adaptive task scheduling for energy efficiency in energy harvesting real-time embedded systems," in Proceedings of the 16th ACM/IEEE International Symposium on Low Power Electronics and Design-ISLPED '10, p. 325, ACM Press, Austin, TX, USA, 2010.

[123] J. Lu, D. Birru, and K. Whitehouse, "Using simple light sensors to achieve smart daylight harvesting," in Proceedings of the 2nd ACM Workshop on Embedded Sensing Systems for Energy-Efficiency in Building-BuildSys '10, p. 73, ACM Press, Zurich, Switzerland, 2010.

[124] C. Moser, J.-J. Chen, and L. Thiele, "An energy management framework for energy harvesting embedded systems," ACM Journal on Emerging Technologies in Computing Systems, vol. 6 , no. 2, pp. 1-21, 2010.

[125] J. R. Piorno, C. Bergonzini, D. Atienza, and T. S. Rosing, "HOLLOWS: a power-aware task scheduler for energy harvesting sensor nodes," Journal of Intelligent Material Systems and Structures, vol. 21, no. 13, pp. 1317-1335, 2010.

[126] G. Waltisperger, C. Condemine, and S. Basrour, "Photovoltaic energy harvester for micro-scale applications," in Proceedings of the 8th IEEE International NEWCAS Conference 2010, Montreal, Canada, 2010.

[127] W. S. Wang, T. O’Donnell, N. Wang, M. Hayes, B. O’Flynn, and C. O'Mathuna, "Design considerations of sub-mW indoor light energy harvesting for wireless sensor systems," ACM Journal on Emerging Technologies in Computing Systems, vol. 6, no. 2, pp. 1-26, 2010.

[128] C. Moser, Power Management in Energy Harvesting Embedded Systems, ETH, Zurich, Switzerland, 2009.

[129] D. Brunelli, D. Dondi, A. Bertacchini, L. Larcher, P. Pavan, and L. Benini, "Photovoltaic scavenging systems: modeling and optimization," Microelectronics Journal, vol. 40, no. 9, pp. 1337-1344, 2009.

[130] D. Brunelli, C. Moser, L. Thiele, and L. Benini, "Design of a solar-harvesting circuit for batteryless embedded systems," IEEE Transactions on Circuits and Systems I: Regular Papers, vol. 56, no. 11, pp. 2519-2528, 2009.
[131] D. Brunelli and L. Benini, "Designing and managing submilliwatt energy harvesting nodes: opportunities and challenges," in Proceedings of the 2009 1st International Conference on Wireless Communication, Vehicular Technology, Information Theory and Aerospace Electronic Systems Technology, Aalborg, Denmark, 2009.

[132] L. Garbuio, M. Lallart, D. Guyomar, C. Richard, and D. Audigier, "Mechanical energy harvester with ultralow threshold rectification based on SSHI nonlinear technique," IEEE Transactions on Industrial Electronics, vol. 56, no. 4, pp. 1048-1056, 2009.

[133] C. Moser, J.-J. Chen, and L. Thiele, "Power management in energy harvesting embedded systems with discrete service levels," in Proceedings of the 14th ACM/IEEE International Symposium on Low Power Electronics and Design-ISLPED '09, p. 413, ACM Press, San Fancisco, CA, USA, 2009.

[134] W. S. Wang, T. O’Donnell, L. Ribetto, B. O’Flynn, M. Hayes, and C. O'Mathuna, "Energy harvesting embedded wireless sensor system for building environment applications," in Proceedings of the 2009 1st International Conference on Wireless Communication, Vehicular Technology, Information Theory and Aerospace Electronic Systems Technology, Aalborg, Denmark, 2009.

[135] D. K. Noh, L. Wang, Y. Yang, H. K. Le, and T. Abdelzaher, "Minimum variance energy allocation for a solar-powered sensor system," in Distributed Computing in Sensor Systems, pp. 44-57, Springer, Berlin, Germany, 2009.

[136] K. Vijayaraghavan and R. Rajamani, "Active control based energy harvesting for battery-less wireless traffic sensors: theory and experiments," in Proceedings of the 2008 American Control Conference, Seattle, WA, USA, 2008.

[137] D. Brunelli, L. Benini, C. Moser, and L. Thiele, "An efficient solar energy harvester for wireless sensor nodes," in Proceedings of the 2008 Design, Automation and Test in Europe, Munich, Germany, 2008.

[138] D. Dondi, A. Bertacchini, L. Larcher, P. Pavan, D. Brunelli, and L. Benini, "A solar energy harvesting circuit for low power applications," in Proceedings of the 2008 IEEE International Conference on Sustainable Energy Technologies, Singapore, 2008.

[139] D. Lee, "Energy harvesting chip and the chip based power supply development for a wireless sensor network," Sensors, vol. 8, no. 12, pp. 7690-7714, 2008.

[140] C. Moser, J.-J. Chen, and L. Thiele, "Reward maximization for embedded systems with renewable energies," in Proceedings of the 2008 14th IEEE International Conference on Embedded and Real-Time Computing Systems and Applications, Kaohsiung, Taiwan, 2008.

[141] G. Park, T. Rosing, M. D. Todd, C. R. Farrar, and W. Hodgkiss, "Energy harvesting for structural health monitoring sensor networks," Journal of Infrastructure Systems, vol. 14, no. 1, pp. 64-79, 2008.

[142] J. T. Scruggs, "Multi-objective optimal control of vibratory energy harvesting systems," in Proceedings of the 15th International Symposium on: Smart Structures and Materials \& Nondestructive Evaluation and Health Monitoring, San Diego, CA, USA, 2008.

[143] S. Chalasani and J. M. Conrad, "A survey of energy harvesting sources for embedded systems," in Proceedings of the IEEE SoutheastCon 2008, Huntsville, AL, USA, 2008.

[144] A. S. Weddell, N. J. Grabham, N. R. Harris, and N. M. White, "Flexible integration of alternative energy sources for autonomous sensing," in Proceedings of the 2008 2nd 
Electronics System-Integration Technology Conference, London, UK, 2008.

[145] H. Chen, C. Jia, C. Zhang, Z. Wang, and C. Liu, "Power harvesting with PZT ceramics," in Proceedings of the 2007 IEEE International Symposium on Circuits and Systems, New Orleans, LA, USA, 2007.

[146] J. Colomer, P. Miribel, A. Saiz-Vela et al., "SiP power management unit with embedded temperature sensor powered by piezoelectric vibration energy harvesting," in Proceedings of the 2007 50th Midwest Symposium on Circuits and Systems, Montreal, Canada, 2007.

[147] A. Kansal, J. Hsu, S. Zahedi, and M. B. Srivastava, "Power management in energy harvesting sensor networks," ACM Transactions on Embedded Computing Systems, vol. 6, no. 4, p. 32, 2007.

[148] K. S. Moon, H. Liang, J. Yi, and B. Mika, "Tire tread deformation sensor and energy harvester development for smart-tire applications," in Proceedings of the 14th International Symposium on: Smart Structures and Materials \& Nondestructive Evaluation and Health Monitoring, San Diego, CA, USA, 2007.

[149] K. Vijayaraghavan and R. Rajamani, "Active control based energy harvesting for battery-less wireless traffic sensors," in Proceedings of the 2007 American Control Conference, New York, NY, USA, 2007.

[150] L. Chao, C. Y. Tsui, and W. H. Ki, "Vibration energy scavenging and management for ultra low power applications," in Proceedings of the 2007 International Symposium on Low Power Electronics and Design-ISLPED '07, pp. 316-321, ACM Press, Portland, OR, USA, 2007.

[151] K. Klues, V. Handziski, C. Lu et al., "Integrating concurrency control and energy management in device drivers," in Proceedings of the 21st ACM SIGOPS Symposium on Operating Systems Principles-SOSP '07, p. 251, 2007.

[152] Y. Cho, N. Chang, C. Chakrabarti, and V. Sarma, "High-level power management of embedded systems with applicationspecific energy cost functions," in Proceedings of the 2006 43rd ACM/IEEE Design Automation Conference, San Francisco, CA, USA, 2006.

[153] V. Raghunathan and P. H. Chou, "Design and power management of energy harvesting embedded systems," in Proceedings of the 2006 International Symposium on Low Power Electronics and Design-ISLPED '06, p. 369, ACM Press, Tegernsee, Germany, 2006.

[154] V. Singhvi, A. Krause, C. Guestrin, J. H. Garrett, and H. S. Matthews, "Intelligent light control using sensor networks," in Proceedings of the 3rd International Conference on Embedded Networked Sensor Systems-SenSys '05, p. 218, ACM Press, San Diego, CA, USA, 2005.

[155] R. Amirtharajah, J. Collier, J. Siebert, B. Zhou, and A. Chandrakasan, "DSPs for energy harvesting sensors: applications and architectures," IEEE Pervasive Computing, vol. 4 , no. 3, pp. 72-79, 2005.

[156] J.-J. Chen, T.-W. Kuo, and C.-S. Shih, “ $1+\varepsilon$ approximation clock rate assignment for periodic real-time tasks on a voltage-scaling processor," in Proceedings of the 5th ACM International Conference on Embedded Software-EMSOFT '05, p. 247, ACM Press, Jersey City, NJ, USA, 2005.

[157] R. Xu, D. Zhu, C. Rusu, R. Melhem, and D. Mossé, "Energyefficient policies for embedded clusters," ACM SIGPLAN Notices, vol. 40, no. 7, p. 1, 2005.

[158] V. Raghunathan, A. Kansal, J. Hsu, J. Friedman, and M. Srivastava, "Design considerations for solar energy harvesting wireless embedded systems," in Proceedings of the 4th International Symposium on Information Processing in Sensor Networks, 2005, Boise, ID, USA, 2005.

[159] J. A. Paradiso and T. Starner, "Energy scavenging for mobile and wireless electronics," IEEE Pervasive Computing, vol. 4, no. 1, pp. 18-27, 2005.

[160] H. El Ghor and M. Chetto, "Energy guarantee scheme for real-time systems with energy harvesting constraints," International Journal of Automation and Computing, vol. 16, no. 3, pp. 354-368, 2019.

[161] S. Liu, Q. Wu, and Q. Qiu, “An adaptive scheduling and voltage/frequency selection algorithm for real-time energy harvesting systems," in Proceedings of the 46th Annual Design Automation Conference on ZZZ-DAC '09, p. 782, ACM Press, San Francisco, CA, USA, 2009.

[162] S. Liu, Q. Qiu, and Q. Wu, "Energy aware dynamic voltage and frequency selection for real-time systems with energy harvesting," in Proceedings of the 2008 Design, Automation and Test in Europe, Munich, Germany, 2008.

[163] AVNET, Powering the Internet of Things via Energy Harvesting, AVNET, Phoenix, AZ, USA, 2017, https://www. avnet.com/wps/portal/us/resources/article/powering-theinternet-of-things-via-energy-harvesting.

[164] W. Zhou, Y. Jia, A. Peng, Y. Zhang, and P. Liu, "The effect of IoT new features on security and privacy: new threats, existing solutions, and challenges yet to be solved," IEEE Internet of Things Journal, vol. 6, no. 2, pp. 1606-1616, 2019.

[165] K. Fu, T. Kohno, D. Lopresti et al., "Safety, security, and privacy threats posed by accelerating trends in the internet of things," 2020, https://arxiv.org/abs/2008.00017.

[166] S. Sicari, A. Rizzardi, L. A. Grieco, and A. Coen-Porisini, "Security, privacy and trust in internet of things: the road ahead," Computer Networks, vol. 76, pp. 146-164, 2015.

[167] Y. Yang, L. Wu, G. Yin, L. Li, and H. Zhao, "A survey on security and privacy issues in internet-of-things," IEEE Internet of Things Journal, vol. 4, no. 5, pp. 1250-1258, 2017. 